OPEN ACCESS

\section{General perturbations for braneworld} compactifications and the six dimensional case

To cite this article: S.L. Parameswaran et al JHEP03(2009)136

View the article online for updates and enhancements.
Related content

- A no-go for no-go theorems prohibiting
$\frac{\text { cosmic acceleration in extra dimensional }}{\text { models }}$
Rik Koster and Marieke Postma
- Self-dual vector multiplet in 3D withgauged
$\frac{\text { Scale covariance }}{\text { Hitoshi Nishino and Subhash Rajpoot }}$
- Chiral zero modes on intersecting heterotic
$\frac{\text { 5-branes }}{\text { Tetsuji Kimura and Shun'ya Mizoguchi }}$

\section{Recent citations}

- U(1) gauge vector field on a codimension-
$\frac{2 \text { brane }}{\text { Chun-E Fu et al }}$
- Gravity in a warped 6D world with an extra
$\frac{\text { DD sphere }}{\text { Akira Kokado and Takesi Saito }}$
- Stability and (quasi)localization of
$\frac{\text { gravitational fluctuations in an Eddington- }}{\text { inspired Born-Infeld brane system }}$
Qi-Ming Fu et al




\title{
General perturbations for braneworld compactifications and the six dimensional case
}

\author{
S.L. Parameswaran, ${ }^{a}$ S. Randjbar-Daemi ${ }^{b}$ and A. Salvio ${ }^{c, d}$ \\ ${ }^{a}$ II Institute for Theoretical Physics, University of Hamburg, \\ DESY Theory Group, Notkestrasse 85, Bldg. 2a, D-22603 Hamburg, Germany \\ ${ }^{b}$ International Center for Theoretical Physics, Strada Costiera 11, 34014 Trieste, Italy \\ ${ }^{c}$ Institut de Théorie des Phénomènes Physiques, EPFL, CH-1015 Lausanne, Switzerland \\ ${ }^{d}$ IFAE, Universitat Autònoma de Barcelona, 08193 Bellaterra, Barcelona, Spain \\ E-mail: susha.louise.parameswaran@desy.de, seif@ictp.trieste.it, \\ salvio@ifae.es
}

ABSTRACT: Our main objective is to study how braneworld models of higher codimension differ from the 5D case and traditional Kaluza-Klein compactifications. We first derive the classical dynamics describing the physical fluctuations in a wide class of models incorporating gravity, non-Abelian gauge fields, the dilaton and two-form potential, as well as 3-brane sources. Next, we use these results to study braneworld compactifications in $6 \mathrm{D}$ supergravity, focusing on the bosonic fields in the minimal model; composed of the supergravity-tensor multiplet and the $\mathrm{U}(1)$ gauge multiplet whose flux supports the compactification. For unwarped models sourced by positive tension branes, a harmonic analysis allows us to solve the large, coupled, differential system completely and obtain the full 4D spin-2,1 and 0 particle spectra, establishing (marginal) stability and a qualitative behaviour similar to the smooth sphere compactification. We also find interesting results for models with negative tension branes; extra massless Kaluza-Klein vector fields can appear in the spectra, beyond those expected from the isometries in the internal space. These fields imply an enhanced gauge symmetry in the low energy 4D effective theory obtained by truncating to the massless sector, which is explicitly broken as higher modes are excited, until the full 6D symmetries are restored far above the Kaluza-Klein scale. Remarkably, the low energy effective theory does not seem to distinguish between a compactification on a smooth sphere and these singular, deformed spheres.

Keywords: Field Theories in Higher Dimensions, Large Extra Dimensions, p-branes, Supergravity Models

ArXiv ePrint: 0902.0375 


\section{Contents}

1 Introduction 1

2 The model 3

2.1 Field content 4

2.2 The action 5

2.3 The equations of motion (EOMs) and solutions 6

3 General perturbations $\quad 9$

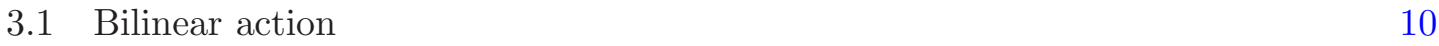

$\begin{array}{lll}3.2 & \text { Local symmetries } & 12\end{array}$

4 Perturbations in the light cone static gauge $\quad \mathbf{1 2}$

$\begin{array}{lll}4.1 & \text { Gauge fixing } & 13\end{array}$

4.2 Bilinear action in the light cone static gauge 13

$\begin{array}{lll}\text { 4.2.1 Spin-2 action } & 14\end{array}$

4.2.2 Spin-1 action $\quad 14$

4.2.3 Spin-0 action and singularities due to backreacting, fluctuating branes 15

$5 \quad 6 D$ (and 5D) braneworlds $\quad 16$

$\begin{array}{ll}5.1 \text { Gravitational fluctuations } & 16\end{array}$

$\begin{array}{lll}\text { 5.1.1 Randall-Sundrum } & 18\end{array}$

$\begin{array}{ll}\text { 5.1.2 6D brane worlds } & 18\end{array}$

$\begin{array}{ll}5.2 \text { Vector fluctuations } & 19\end{array}$

$\begin{array}{ll}\text { 5.2.1 Randall-Sundrum } & 20\end{array}$

$\begin{array}{ll}\text { 5.2.2 } 6 \mathrm{D} \text { brane worlds } & 20\end{array}$

5.3 Massless vectors and 4D gauge symmetries 26

$\begin{array}{ll}\text { 5.3.1 Why there are three massless vector modes } & 27\end{array}$

5.3.2 The absence of enhanced gauge symmetries in the full 4D theory 27

5.3.3 The emergence of enhanced gauge symmetries at low energies 29

5.4 Massless scalars 30

$\begin{array}{lll}\text { 5.4.1 Randall-Sundrum } & 30\end{array}$

$\begin{array}{lll}5.4 .2 & 6 \mathrm{D} \text { brane worlds } & 31\end{array}$

$\begin{array}{lll}6 & \text { Summary of results } & 31\end{array}$

$\begin{array}{lll}7 & \text { Conclusions } & 33\end{array}$

A General $\xi$-dependent bilinear action 35

B Spin-0 bilinear action in the light cone static gauge 36 


\section{Introduction}

Almost two decades on, branes are evermore ubiquitous in the models constructed to understand particle physics and cosmology, with all their How?'s and Why?'s. As fundamental objects, they are the D-branes and NS-branes (or M-branes) of string (or M) theory, but within a low-energy effective field theory description, they are introduced as braneworlds. Often these braneworlds are considered as infinitely thin but finite tension objects, like for their more fundamental cousins, although sometimes it proves necessary to resolve their structure by adding some thickness.

A codimension one brane necessarily forms a boundary in the bulk space, since there is no path which can lead from one side to the other without traversing the brane. The gravitational backreaction of these objects is well understood; whilst the metric is continuous across the brane, its first derivative can have finite discontinuities. Branes with more than one transverse dimension are qualitatively different, and much harder, due to their sourcing of singularities in the transverse space. Still, codimension two branes can also be handled with some control; they backreact on the geometry in such a way as to produce relatively mild conical singularities.

The construction of solutions sourced by branes, with up to two codimensions, in various field theory models is by now a well-developed art. In 5D, the archetype is of course the construction of Randall and Sundrum [1,2]. In 6D, we take the general warped braneworld compactifications ("conical-GGP solutions") of 6D N=1 gauged supergravity [3] found in $[4,5]$ as representative. These solutions additionally invoke fluxes, which are also playing a dominant role in string compactifications today, and indeed models with two extra dimensions are the simplest in which flux compactifications can be studied. Having established the solutions, we can begin to ask about their physics: Are they stable to small perturbations? What are the symmetries and particle content of the low energy effective field theory? Is it chiral? What are the modifications to 4D Einsteinian gravity? What would be the effective vacuum energy measured by a $4 \mathrm{D}$ observer? What role do the branes play in these and other phenomena? And so on.

The first step towards answering these questions is to analyze the classical spectra of small fluctuations around the solution. A number of such studies have been made recently for the conical-GGP solutions. In [6] we worked out the spectra for certain $4 \mathrm{D}$ gauge fields and fermions present in the model and no tachyons or ghosts were found amongst them. A similar (marginal) stability was found in [7], where the axially symmetric modes for some of the scalar perturbations were calculated. The spectrum for the gravitino has also been analyzed in [8]. In [9], meanwhile, we studied the tachyonic instabilities that can arise from the non-axially symmetric, 4D scalar fluctuations descending from $6 \mathrm{D}$ gauge fields, 
and charged under the background fluxes. ${ }^{1}$ Whether a given model with a given flux suffers from this instability turns out to depend on the tensions of the branes present.

We now intend to complete the spectral analysis for the bosonic fluctuations about the braneworld solutions of 6D supergravity. Our particular focus in this paper is on the so-called Salam-Sezgin sector - that arising from the supergravity-tensor multiplet and the U(1) gauge multiplet in which the background monopole lies - which was partially treated in $[7,11]$. The remaining sectors have been completed elsewhere [6, 9]. We will calculate the corresponding spectra for the $4 \mathrm{D}$ spin-2 and - for unwarped backgrounds spin- 1 and spin-0 fields. The model that we are studying is complicated, and technically difficult. However, this goes hand in hand with its advantage of generality, and indeed the results for several simpler scenarios can be extracted from our work at its various stages.

Our approach will be that established in [12], where a formalism was developed to analyze the spectra of small perturbations about arbitrary solutions of Einstein, YangMills and scalar systems. The first part of this paper can be considered as a generalization of that work, where we now include the presence of thin source 3-branes and extra bulk fields that are generically present in supergravity theories; the dilaton and anti-symmetric two-form potential. With little extra cost, we actually keep the number of dimensions transverse to the brane general.

We first derive the general form of the bilinear action that describes the behaviour of small fluctuations. For codimension-two or higher, we include fluctuations of the brane positions in the transverse directions, the so-called "branons". We then apply the lightcone gauge (for bulk fields) and static gauge (for branons) to restrict to physical degrees of freedom, and decouple the dynamics for the spin-2, -1 and -0 fluctuations. The gauge-fixed bilinear action thus obtained provides the starting point to calculate the Kaluza-Klein (KK) spectra for the conical-GGP solutions, as well as, for example, the 5D Randall-Sundrum models and the non-supersymmetric Einstein-Yang Mills(-dilaton) model in any dimension.

In the second part of this paper, we use these general equations to study the behaviour of braneworld models in 6D (along the way also recover some of well-known aspects of the $5 \mathrm{D}$ scenarios). Here, since we include the backreaction of the branes, the dynamics of the branons are not well-defined. ${ }^{2}$ Therefore, to study the spin-0 sector, we choose to truncate the branons by e.g. placing the branes at orbifold fixed points, or taking the brane tensions to be very large making the branes rigid within our range of validity. Meanwhile, the conical singularities in the curvature that are induced by the codimension two branes do not prevent us from understanding the behaviour of the bulk fluctuations.

We are able to derive the spectrum for the $4 \mathrm{D}$ spin-2 fields in the model's full warped generality. The spin-1 and spin-0 sectors present large coupled differential systems, and by finding a set of harmonics on the 2D internal space ( the "rugbyball"), we are also able to solve these systems analytically for the unwarped case. In this way, we obtain all the 4D modes for unwarped compactifications with positive tension brane sources, and

\footnotetext{
${ }^{1}$ The end point of this instability is studied in [10].

${ }^{2}$ Indeed, the behaviour of the branons is usually considered under the probe brane approximation, in which the brane tension is much smaller than the bulk gravitational scale, so that the backreaction can be neglected [13, 14].
} 
qualitatively, we observe the same behaviour as in the smooth sphere compactification without branes — including marginal stability.

In the presence of negative tension codimension-two branes, meanwhile, the physics can surprise. Here, despite the fact that brane sources clearly break the $\mathrm{SU}(2)$ isometries of the sphere to U(1), three massless spin-1 fields ${ }^{3}$ can be found amongst the KK spectra for special values of the conical deficit angle. These special deficit angles, $\delta=-2 \pi,-4 \pi, \ldots$, allow three Killing vectors to be well-defined everywhere outside the branes, although only one of them can be globally integrated to an isometry.

Whether or not the massless vectors are gauge bosons of an enhanced gauge symmetry in the $4 \mathrm{D}$ theory can be understood by going beyond bilinear order and considering the interaction terms. We find the presence of KK modes that are not in well-defined representations of the $\mathrm{SU}(2)$ generated by the Killing vectors, and therefore the full $4 \mathrm{D}$ theory does not enjoy an $\mathrm{SU}(2)$ gauge symmetry. For this reason, we do not expect the classical masslessness of the vector fields to survive quantum corrections. Meanwhile, all our bosonic massless modes do fall into well-defined SU(2) representations, and therefore we argue that the classical low energy $4 \mathrm{D}$ effective field theory - obtained by truncating to the massless sector - does enjoy an enhanced KK gauge symmetry beyond the isometries! Moreover, it appears that the low energy theory does not distinguish between compactifications on the smooth sphere and these singular, deformed spheres.

Let us now give an outline for the remainder of the paper. The first part presents a rather general analysis that determines the dynamics of perturbations in braneworld compactifications. In the next section, we introduce the model (both theories and background solutions) and discuss the scenarios to which our analysis can be applied. In section 3 , we introduce the perturbations about the background, obtain the bilinear action that describes their dynamics, and discuss the local symmetries of this action. In section 4 , we use these symmetries to fix to the "light cone static gauge", and give the bilinear action in this gauge, in which the different spin sectors decouple.

Then begins the second part, which uses the previous results to study the $4 \mathrm{D}$ fields that emerge in various scenarios. In section 5, our main interest is in the braneworld solutions of $6 \mathrm{D}$ supergravity, but we also discuss a non-supersymmetric $6 \mathrm{D}$ model and the 5D Randall-Sundrum models. In the main text we present the KK spectra for spin-2 and spin-1 fields and identify the massless spin-0 fields; the complete spin-0 sector can be found in the appendices. Finally, we understand in detail the physical significance of the extra massless $4 \mathrm{D}$ vector modes that can appear in the spectra, and the gauge invariance that emerges in the $4 \mathrm{D}$ theory.

We summarise our results in section 6 , before concluding in section 7 .

\section{The model}

We begin with the definition of our model. The main focus of the present paper will be a class of bosonic $6 \mathrm{D}$ field theories with thin codimension-two branes. In particular we are

\footnotetext{
${ }^{3}$ In addition to any massless gauge fields arising from unbroken higher dimensional gauge symmetries.
} 
interested in the bosonic part of $6 \mathrm{D} \mathrm{N}=1$ gauged supergravity [3]. However, throughout the article we shall keep a general space-time dimension $D$ as far as possible, and certain truncations of the field content allow our analysis to be applied to several different scenarios, including the non-supersymmetric Einstein-Yang-Mills theory or the RandallSundrum Model.

\section{$2.1 \quad$ Field content}

The basic ingredients of our model are the higher dimensional metric $G_{M N}$, where the space-time indices run over $M, N, \ldots=0, \ldots, D-1$, and the gauge field $\mathcal{A}_{M}$ of a compact Lie group $\mathcal{G}$. These are bulk fields in the sense that they depend on all the space-time coordinates $X^{M}$.

We also want to consider a certain number $\mathcal{N}$ of 3 -branes embedded in the $D$ dimensional space time. To do so we introduce, following ref. [15], $\mathcal{N}$ functions $Y_{k}^{M}\left(x_{k}\right), k=$ $1, \ldots, \mathcal{N}$, which represent the positions of the branes in the $D$-dimensional space time. The $x_{k}$ represent the $4 \mathrm{D}$ coordinates on the brane, $x_{k}=\left\{x_{k}^{\alpha}\right\}$, where $\alpha, \beta, \ldots$ are the $4 \mathrm{D}$ indices. Not all the space-time components of $Y_{k}^{M}\left(x_{k}\right)$ are physical degrees of freedom: 4 space-time components for each $k$ can be gauged away by using the $4 \mathrm{D}$ (general) coordinate transformation invariance acting on $x_{k}$ [15], as we will explicitly do in Subsection 4.1. We consider $Y_{k}^{M}\left(x_{k}\right)$ to be a brane field because it depends only on a $4 \mathrm{D}$ world-volume coordinate. These fields are important to introduce the branes in a covariant way, and indeed we can construct the induced metrics on the branes by means of

$$
g_{\alpha \beta}^{k}=G_{M N}\left(Y_{k}\left(x_{k}\right)\right) \partial_{\alpha} Y_{k}^{M}\left(x_{k}\right) \partial_{\beta} Y^{M}\left(x_{k}\right) .
$$

In order to complete the bosonic part of the $6 \mathrm{D}$ supergravity, one should add other bulk fields in addition to $G_{M N}$ and $\mathcal{A}_{M}$, that is a dilaton $\phi$ and a 2-form field $B_{M N}$, which emerge from the graviton multiplet and an antisymmetric tensor multiplet [3]. We will refer to $B_{M N}$ as the Kalb-Ramond field. Moreover, concerning the $6 \mathrm{D}$ supergravity, we shall assume that $\mathcal{G}$ is a product of simple groups that include a $\mathrm{U}(1)_{R}$ gauged R-symmetry. In general one can also add some hypermultiplets [3], which turn out to be important to cancel gauge and gravitational anomalies $[16,17]$. In the bosonic sector this leads to additional scalar fields $\Phi^{\alpha}$ (hyperscalars) in some representation of $\mathcal{G}$; however, from now on we set $\Phi^{\alpha}=0$. We do so because we are interested in the linear perturbations which mix with the $D$-dimensional gravitational fluctuations $h_{M N}$ : indeed, for the class of backgrounds we are interested in (see Subsection 2.3), the $\Phi^{\alpha}$ decouple from $h_{M N}$. Their inclusion should be straightforward.

Therefore the bulk and the brane field contents that we consider are respectively:

$$
\left\{G_{M N}, \mathcal{A}_{M}, \phi, B_{M N}\right\} \quad \text { and } \quad\left\{Y_{k}^{M}\left(x_{k}\right), \ldots\right\}
$$

The dots in the second set of (2.2) represent additional brane fields that we can always introduce, but which are not required by general covariance; for example they can be the fields of the Standard Model (SM). 


\subsection{The action}

We split the action functional $S$ into the bulk action $S_{B}$, which depends only on the bulk fields, and the brane action $S_{b}$ that is a functional of the brane fields as well.

The bulk action is ${ }^{4}[3]$

$$
S_{B}=\int d^{D} X \sqrt{-G}\left\{\frac{1}{\kappa^{2}}\left[R-\frac{1}{4}(\partial \phi)^{2}\right]-\frac{1}{4} e^{\phi / 2} F^{2}-\frac{\kappa^{2}}{48} e^{\phi} H_{M N P} H^{M N P}-\mathcal{V}(\phi)\right\}
$$

where $G$ is the determinant of $G_{M N}$ and $\kappa$ is the $D$-dimensional Planck scale; also ${ }^{5} F^{2} \equiv$ $F_{M N} F^{M N}$ and $(\partial \phi)^{2} \equiv \partial_{M} \phi \partial^{M} \phi$. The explicit expression for the gauge field strength $F_{M N}$ is ${ }^{6}$

$$
F_{M N}=\partial_{M} \mathcal{A}_{N}-\partial_{N} \mathcal{A}_{M}+g \mathcal{A}_{M} \times \mathcal{A}_{N},
$$

where $g$ is the gauge coupling, which in fact represents a collection of independent gauge couplings including that of the $\mathrm{U}(1)_{R}$ subgroup, $g_{1} . H_{M N P}$ is the Kalb-Ramond field strength, which contains a Chern-Simons coupling as follows [18]:

$$
H_{M N P}=\partial_{M} B_{N P}+F_{M N} \mathcal{A}_{P}-\frac{g}{3} \mathcal{A}_{M}\left(\mathcal{A}_{N} \times \mathcal{A}_{P}\right)+2 \text { cyclic perms } .
$$

The function $\mathcal{V}(\phi)$ is the dilaton potential. In the supersymmetric model this is fixed to be $\mathcal{V}(\phi)=8 g_{1}^{2} e^{-\phi / 2} / \kappa^{4}$.

Meanwhile, we consider the following 3-brane action

$$
S_{b}=\sum_{k}\left(-T_{k} \int d^{4} x_{k} \sqrt{-g^{k}}\right) \equiv-T \int d^{4} x \sqrt{-g}
$$

where $g^{k}$ is the determinant of (2.1) and $T_{k}$ are the tensions of the branes. From now on (unless otherwise stated) we suppress the index $k$, as we have done on the right hand side of (2.6). The reader may have noticed that we have not introduced the Gibbons-Hawking boundary term, which is generically necessary to treat codimension one branes [19]. Indeed, we shall apply our analysis only to those codimension one models whose branes are placed on orbifold fixed points, in which case the Gibbons-Hawking boundary term is not present [20].

We can summarise by saying that our analysis will apply to the following two types of models:

1. $6 \mathrm{D} \mathrm{N}=1$ gauged supergravity.

2. Einstein-Yang-Mills theories, with a dilaton or cosmological constant $\Lambda$, for a general space-time dimension.

\footnotetext{
${ }^{4}$ We choose signature $(-,+, \ldots,+)$, and define $R_{M N}{ }^{R}{ }_{S}=\partial_{M} \Gamma_{N S}^{R}-\partial_{N} \Gamma_{M S}^{R}+\Gamma_{M P}^{R} \Gamma_{N S}^{P}-\Gamma_{N P}^{R} \Gamma_{M S}^{P}$ and $R_{M N}=R_{P M}{ }^{P}{ }_{N}$.

${ }^{5} \mathrm{~A}$ trace overall is understood when we write a product of Lie algebra valued objects: e.g. in eq. (2.3) $F^{2} \equiv \operatorname{Tr}\left(F^{2}\right)$.

${ }^{6}$ We define the cross-product as $\left(\mathcal{A}_{M} \times \mathcal{A}_{N}\right)^{I}=f^{I J K} \mathcal{A}_{M}^{J} \mathcal{A}_{N}^{K}$, with $f^{I J K}$ the structure constants of $\mathcal{G}$ : $\left[T^{I}, T^{J}\right]=i f^{I J K} T^{K}$, where $T^{I}$ are the generators of $\mathcal{G}$.
} 
The second case includes, for example, the RS models $[1,2]$ or the non-supersymmetric $6 \mathrm{D}$ Einstein-Yang-Mills- $\Lambda$ (EYM $\Lambda)$ model $[13,21]$. They can be obtained by simply fixing the appropriate dimension and setting $H_{M N P}=0, \phi=0$ and $\mathcal{V}(0)=\Lambda$. Even if our main interest is in models of Type 1 we will also consider the second class for several reasons. In this way, we will see that our results can be applied in quite general contexts, and it will also provide interesting additional ways to check our formulae. Moreover, in the future it should help us to figure out the role of supersymmetry in the linear perturbations.

Finally, it is important to note that the actions $S_{B}$ and $S_{b}$ are invariant with respect to both the $D$-dimensional and the $4 \mathrm{D}$ coordinate transformations (acting respectively on $X^{M}$ and $x^{\alpha}$ ). We will discuss the local symmetries of the present model and an explicit gauge fixing for the linear perturbations in Subsections 3.2 and 4.1.

\subsection{The equations of motion (EOMs) and solutions}

The EOMs that follow from the variation of the action $S_{B}+S_{b}$ are:

$$
\begin{aligned}
R^{M N}-\frac{1}{2} G^{M N} R & =\frac{\kappa^{2}}{2}\left\{e^{\phi / 2}\left(F_{P}^{M} F^{N P}-\frac{1}{4} G^{M N} F^{2}\right)+\frac{1}{2 \kappa^{2}} \partial^{M} \phi \partial^{N} \phi\right. \\
\left.-G^{M N}\left[\frac{1}{4 \kappa^{2}}(\partial \phi)^{2}+\mathcal{V}(\phi)\right]\right\}-T \kappa^{2} \mathcal{B}^{M N} & \\
D_{N}\left(e^{\phi / 2} F^{N M}\right) & =0 \\
\frac{1}{2 \kappa^{2}} D^{2} \phi & =\frac{\partial \mathcal{V}}{\partial \phi}(\phi)+\frac{1}{8} e^{\phi / 2} F^{2} \\
\frac{1}{\sqrt{-g}} \partial_{\alpha}\left(\sqrt{-g} G_{M N} \partial^{\alpha} Y^{N}\right) & =\frac{1}{2} \partial_{M} G_{N P} \partial Y^{N} \cdot \partial Y^{P}
\end{aligned}
$$

where we have fixed $H_{M N P}=0$, since our interest shall be in backgrounds that enjoy $4 \mathrm{D}$ Poincaré invariance. Moreover, in eq. (2.9) and (2.10) we have introduced the notation $D^{2} \phi \equiv D_{M} D^{M} \phi$, where $D_{M}$ is the covariant derivative, and $\partial Y^{M} \cdot \partial Y^{N} \equiv \partial_{\alpha} Y^{M} \partial^{\alpha} Y^{N}$. Recall also that we have suppressed the index $k$ on $Y_{k}^{M}$, which labels each of the branes. The last term in (2.7) represents the brane contribution to the Einstein equations, where $\mathcal{B}^{M N}$ is defined by

$$
\mathcal{B}^{M N}(X) \equiv \frac{1}{2} \int d^{4} x \sqrt{g / G} \delta(X-Y(x)) \partial Y^{M} \cdot \partial Y^{N}
$$

we note that the bulk quantity $G$ in $(2.11)$ is computed at the position of the brane $(G=G(Y))$ because of the presence of the $D$-dimensional delta function $\delta(X-Y(x))$. Furthermore, since eqs. (2.10) come from the variation of the brane action with respect to $Y^{M}$, there the bulk fields $G_{M N}$ and $\partial_{M} G_{N P}$ are computed at the brane position $\left(G_{M N}=\right.$ $G_{M N}(Y)$ and $\left.\partial_{M} G_{N P}=\partial_{M} G_{N P}(Y)\right)$.

In the present paper we will focus mainly on the following ansatz solution 
to $(2.7)-(2.10)$ :

$$
\begin{aligned}
Y^{\mu} & =x^{\mu}, \\
Y^{\underline{m}} & =\text { constant }, \\
d s^{2} & =e^{A(\rho)} \eta_{\mu \nu} d x^{\mu} d x^{\nu}+d \rho^{2}+e^{B(\rho)} K_{m n}(y) d y^{m} d y^{n}, \\
\mathcal{A} & =\mathcal{A}_{m}(\rho, y) d y^{m}, \\
\phi & =\phi(\rho), \\
H_{M N P} & =0,
\end{aligned}
$$

where $\mu=0,1,2,3, m=5, \ldots, 4+D_{2}, \underline{m}=(\rho, m)$ (we have $D=5+D_{2}$ ) and $y^{m}$ and $K_{m n}$ are respectively the coordinate and the metric on the $D_{2}$-dimensional space. Eq. (2.12) is not really an assumption because we can always use the $4 \mathrm{D}$ general coordinate invariance on the branes to set (2.12). Eq. (2.13) is instead a non trivial assumption. Moreover, in Eqs (2.14)-(2.17) we are assuming that the bulk field background has a 4D Poincaré invariance and that the functions $A, B$ and $\phi$ depend only on the coordinate $\rho$. We will also assume $\mathcal{A}$ to lie in the Cartan subalgebra of $\operatorname{Lie}(\mathcal{G})$.

One of the simplest models that can be described by this set up is the Randall-Sundrum (RS) model [1], where we have $D=5, \phi=0$ and $\mathcal{A}=0$ and the internal space is $S^{1} / Z_{2}$ with two branes on the fixed points of $Z_{2}$, say at $\rho=0$ and $\rho=\pi r_{c}$. The explicit form of the solution is given by

$$
A=-2 k|\rho|, \quad Y_{1}^{\rho}=0, \quad Y_{2}^{\rho}=\pi r_{c},
$$

where $k$ is a positive constant. The object $|\rho|$ in (2.18) is equal to the absolute value of $\rho$ in the region $-\pi r_{c}<\rho<\pi r_{c}$ and its value anywhere else is obtained by periodicity. In order for (2.18) to be a solution one needs $T_{1}=-T_{2}=12 k / \kappa^{2}$ and $\Lambda=-12 k^{2} / \kappa^{2}$. In section 5 , we shall use this very well-known solution to check the result given in section 4 .

However, in this paper our main interest lies in the analysis of a class of solutions found by Gibbons, Güven and Pope (GGP) [4] to the 6D supergravity: the general set of warped solutions with 4D Poincaré symmetry, and axial symmetry in the transverse dimensions. Here we give only a subset of this general class, namely that which contains singularities no worse than conical and therefore can be sourced by brane terms of the form (2.6).

To give the explicit expression of the conical-GGP solutions, it turns out to be useful to introduce the following radial coordinate [6]

$$
u(\rho) \equiv \int_{0}^{\rho} d \rho^{\prime} e^{-A\left(\rho^{\prime}\right) / 2}
$$

whose range is $0 \leq u \leq \bar{u} \equiv \pi r_{0} / 2$. In this frame the metric reads

$$
d s^{2}=e^{A(u)}\left(\eta_{\mu \nu} d x^{\mu} d x^{\nu}+d u^{2}\right)+e^{B(u)} \frac{r_{0}^{2}}{4} d \varphi^{2} .
$$


The explicit conical-GGP solutions ${ }^{7}$ are then the following particular case of the ansatz (2.12)-(2.17) [4]:

$$
\begin{aligned}
e^{A} & =e^{\phi / 2}=\sqrt{\frac{f_{1}}{f_{0}}}, \quad e^{B}=4 \alpha^{2} e^{A} \frac{\cot ^{2}\left(u / r_{0}\right)}{f_{1}^{2}}, \\
\mathcal{A} & =-\frac{4 \alpha}{q \kappa f_{1}} Q d \varphi
\end{aligned}
$$

where $q$ and $\alpha$ are generic real numbers and $Q$ is a generator of a $\mathrm{U}(1)$ subgroup of a simple factor of $\mathcal{G}$, satisfying $\operatorname{Tr}\left(Q^{2}\right)=1$. Also,

$$
f_{0} \equiv 1+\cot ^{2}\left(\frac{u}{r_{0}}\right), \quad f_{1} \equiv 1+\frac{r_{0}^{2}}{r_{1}^{2}} \cot ^{2}\left(\frac{u}{r_{0}}\right)
$$

with $r_{0}^{2} \equiv \kappa^{2} /\left(2 g_{1}^{2}\right)$ and $r_{1}^{2} \equiv 8 / q^{2}$.

This solution is supported by two branes located at $u=0$ and $u=\bar{u}$. Indeed, as $u \rightarrow 0$ or $u \rightarrow \bar{u}$, the metric tends to that of a cone, with respective deficit angles

$$
\delta=2 \pi\left(1-|\alpha| \frac{r_{1}^{2}}{r_{0}^{2}}\right) \quad \text { and } \quad \bar{\delta}=2 \pi(1-|\alpha|),
$$

and corresponding delta-function behaviours in the Ricci scalar. We will take $\alpha \geq 0$ without loss of generality. The tensions of the two branes $T$ and $\bar{T}$ are related to the deficit angle as follows [22]:

$$
T=2 \delta / \kappa^{2} \text { and } \bar{T}=2 \bar{\delta} / \kappa^{2} .
$$

Unlike the RS solution, here the warp factor $e^{A}$ is smooth on the brane positions $u=0$ and $u=\bar{u}$. In particular we have

$$
e^{A} \stackrel{u \rightarrow 0, \bar{u}}{\rightarrow} \text { constant } \neq 0, \quad \partial_{u} e^{A} \stackrel{u \rightarrow 0, \bar{u}}{\longrightarrow} 0 .
$$

By using (2.25), (2.12) and (2.13), it is also easy to check that the conical-GGP configuration satisfies the $Y$-equations (2.10) in addition to the bulk EOMs (2.7)-(2.9).

The expression for the gauge field background in eq. (2.21) is well-defined in the limit $u \rightarrow 0$, but not as $u \rightarrow \bar{u}$. We should therefore use a different patch to describe the $u=\bar{u}$ brane, and this must be related to the patch including the $u=0$ brane by a singlevalued gauge transformation. This leads to a Dirac quantization condition, which for a field interacting with $\mathcal{A}$ through a charge $e$ gives

$$
-e \frac{4 \alpha \bar{g}}{\kappa q}=-e \alpha \frac{r_{1}}{r_{0}} \frac{\bar{g}}{g_{1}}=N,
$$

where $N$ is an integer that is called monopole number and $\bar{g}$ is the gauge coupling constant corresponding to the background gauge field. For example, if $\mathcal{A}$ lies in $\mathrm{U}(1)_{R}$, then $\bar{g}=g_{1}$. The charge $e$ can be computed once we have selected the background gauge group, since it is an eigenvalue of the generator $Q$. Also, note that the internal space corresponding to Solutions (2.21) has an $S^{2}$ topology (its Euler number equals 2).

\footnotetext{
${ }^{7}$ The coordinate $u$ is related to the coordinate $r$ in [4] by $r=r_{0} \cot \left(u / r_{0}\right)$.
} 
Finally, we observe that one can obtain the unwarped "rugbyball" compactification [21] simply by setting $r_{0}=r_{1}$. In this case the metric is

$$
d s^{2}=\eta_{\mu \nu} d x^{\mu} d x^{\nu}+\frac{r_{0}^{2}}{4}\left(d \theta^{2}+\alpha^{2} \sin ^{2} \theta d \varphi^{2}\right),
$$

where $\theta \equiv 2 u / r_{0}$, and the background value of the dilaton is zero; therefore this is a solution also to the non-supersymmetric 6D EYM $\Lambda$ model. For $\alpha<1$ the deficit angle is positive. The geometry is also well-defined when $\alpha>1$ and the deficit angle is negative; we name these spaces "saddle-spheres" (see [9] for a detailed discussion on their properties). Moreover, we can smoothly retrieve the sphere compactification (with radius $r_{0} / 2$ ) by taking $\alpha=1$ in addition to $r_{0}=r_{1}$.

\section{General perturbations}

The main purpose of this paper is to study the linear perturbations in the above models. We therefore perturb the fields in (2.2) as follows:

$$
\begin{array}{ll}
G_{M N} \rightarrow G_{M N}+h_{M N}, & \mathcal{A}_{M} \rightarrow \mathcal{A}_{M}+V_{M}, \quad \phi \rightarrow \phi+\tau, \\
B_{M N} \rightarrow B_{M N}+b_{M N}, & Y^{M} \rightarrow Y^{M}+\xi^{M} .
\end{array}
$$

The first terms in the right hand sides of (3.1) represent the background quantities of the corresponding fields. In fact, it is useful to introduce another 2-form field $V_{M N}$ in order to describe the fluctuations of the Kalb-Ramond field. This can be done as follows. Since $H_{M N P}$ appears only quadratically in (2.3), and $H_{M N P}=0$ at the background level due to 4D Poincaré invariance, the linear approximation (which corresponds to the bilinear level in the action) involves only the linear perturbation of $H_{M N P}$, that we denote with $^{8} H_{M N P}^{(1)}$,

$$
H_{M N P}^{(1)}=\left[d\left(b_{2}-\mathcal{A} \wedge V\right)+2 F \wedge V\right]_{M N P},
$$

where we have used the notation of p-forms and $b_{2}$ is the fluctuation in the Kalb-Ramond 2 -form, $\mathcal{A}$ and $F$ the background values of the gauge field and its field strength respectively and $V$ the perturbation of the gauge field. We now introduce the 2-form $V_{2}$ as follows:

$$
V_{2} \equiv \kappa\left(b_{2}-\mathcal{A} \wedge V\right),
$$

whose components will be denoted by $V_{M N} . H_{M N P}^{(1)}$ can now be expressed in terms of $V_{2}$ and $V$ :

$$
H_{M N P}^{(1)}=\left(\frac{1}{\kappa} d V_{2}+2 \gamma F \wedge V\right)_{M N P},
$$

where we have introduced a new parameter $\gamma$; for $\gamma=1$ we recover the structure of $H_{M N P}^{(1)}$ required by the $6 \mathrm{D}$ supergravity, whereas for $\gamma=0$ the fluctuations of $V_{M N}$ are completely decoupled (at the linear level) from the rest. This will allow us to treat simultaneously the $6 \mathrm{D}$ supergravity and the EYM $\Lambda$ models.

Finally, we note that the fields $\xi^{M}(x)$ describe the fluctuations of the brane positions, and as such they are 4D fields.

\footnotetext{
${ }^{8}$ Since the background $H_{M N P}=0$, and the background monopole, $\mathcal{A}$, lies in the Cartan subalgebra, we see that the exterior derivative acting on the background Kalb-Ramond potential $B_{2}$ must be zero. Also, $\mathcal{A} \wedge \mathcal{A}=0$.
} 


\subsection{Bilinear action}

Here we provide the linearized theory which corresponds to the bilinear approximation in the action. The bilinear action has been computed by considering the variation of $S_{B}+S_{b}$ under (3.1) and by keeping only terms up to the quadratic order. ${ }^{9}$ We split it into different contributions as follows:

$$
\begin{aligned}
S(h, h)+S(V, V) & +S(h, V)+S(\tau, \tau)+S(h, \tau)+S(V, \tau) \\
& +S\left(V_{2}, V_{2}\right)+S\left(V, V_{2}\right)+S(\xi, \xi)+S(h, \xi),
\end{aligned}
$$

where $S(h, h)$ is the bilinear action that depends only on the fluctuations $h_{M N}, S(h, V)$ represents the mixing term between $h_{M N}$ and $V_{M}$ and so on. We have $S\left(h, V_{2}\right)=S\left(\tau, V_{2}\right)=$ 0 as a consequence of our background ansatz, for which $H_{M N P}=0$. We give here the explicit expressions for the bilinear action that depend only on the bulk fields; the dynamics of the $\xi^{M}$ fields, are explicitly given in appendix A. We find:

$$
\begin{aligned}
S(h, h)=\int d^{D} X \sqrt{-G} & \left\{\frac{1}{2 \kappa^{2}}\left[\left(h_{; M}^{M N}-\frac{1}{2} h^{; N}\right)^{2}-\frac{1}{2} h_{; M}^{N P} h_{N P} ; M+\frac{1}{4} h^{; M} h_{; M}-\frac{1}{2} R_{1} h^{2}\right]\right. \\
& -\frac{1}{2} h_{P M} h^{P}{ }_{N}\left(\frac{1}{2} e^{\phi / 2} F^{M R} F^{N}{ }_{R}+\frac{1}{4 \kappa^{2}} \partial^{M} \phi \partial^{N} \phi\right) \\
& -\frac{1}{2} h^{M N} h^{P R}\left(\frac{1}{\kappa^{2}} R_{P M N R}-\frac{1}{2} e^{\phi / 2} F_{P M} F_{N R}\right) \\
& \left.-\frac{T}{2}\left[\mathcal{B}^{M N}\left(h_{P M} h^{P}{ }_{N}-h h_{M N}\right)+\frac{1}{2} \mathcal{B}^{M N P R} h_{M N} h_{P R}\right]\right\},
\end{aligned}
$$

where the semicolon denotes the (background) gravitational covariant derivative, $h \equiv$ $G^{M N} h_{M N}, R_{M N}^{P}$ is the Riemann tensor for the background metric and we have defined

$$
\frac{2}{\kappa^{2}} R_{1} \equiv \frac{1}{\kappa^{2}} R-\frac{1}{4} e^{\phi / 2} F^{2}-\frac{1}{4 \kappa^{2}}(\partial \phi)^{2}-\mathcal{V}(\phi)
$$

and

$$
\begin{aligned}
\mathcal{B}^{M N P R} \equiv \int d^{4} x \sqrt{g / G} \delta(X-Y(x))\left[\frac{1}{2}\left(\partial Y^{M} \cdot \partial Y^{N}\right) \partial Y^{P} \cdot \partial Y^{R}\right. & \\
& \left.-\left(\partial Y^{M} \cdot \partial Y^{P}\right) \partial Y^{N} \cdot \partial Y^{R}\right] .
\end{aligned}
$$

The term proportional to $\mathcal{B}^{M N P R}$ in the last line of (3.6) is the contribution to $S(h, h)$ coming from the brane action $S_{b}$, whereas the term proportional to $\mathcal{B}^{M N}$ comes from the

\footnotetext{
${ }^{9}$ The EOMs (2.7)-(2.10) guarantee that the linear terms vanish.
} 
EOMs (2.7), which we have used to write $S(h, h)$ in the form (3.6). Moreover,

$$
\begin{aligned}
& S(V, V)=\int d^{D} X \sqrt{-G}[-\frac{1}{2} e^{\phi / 2}\left(D_{M} V_{N} D^{M} V^{N}-D_{M} V_{N} D^{N} V^{M}\right) \\
&\left.-\frac{\kappa^{2}}{12} \gamma^{2} e^{\phi}\left(F_{[M N} V_{P]}\right)\left(F^{[M N} V^{P]}\right)-\frac{1}{2} \bar{g} e^{\phi / 2} F^{M N} V_{M} \times V_{N}\right] \\
& S(h, V)=-\int d^{D} X \sqrt{-G} e^{\phi / 2}\left(D^{M} V^{N}-D^{N} V^{M}\right)\left(\frac{1}{4} h F_{M N}+h_{P N} F_{M}^{P}\right) \\
& S(\tau, \tau)=-\int d^{D} X \sqrt{-G}\left[\frac{1}{4 \kappa^{2}}(\partial \tau)^{2}+\frac{1}{2} \frac{\partial^{2} \mathcal{V}}{\partial \phi^{2}} \tau^{2}+\frac{1}{32} e^{\phi / 2} F^{2} \tau^{2}\right] \\
& S(h, \tau)=\int d^{D} X \sqrt{-G}\left[\frac{1}{2 \kappa^{2}} \partial_{M} \tau \partial_{N} \phi\left(h^{M N}-\frac{1}{2} G^{M N} h\right)-\frac{1}{2} \frac{\partial \mathcal{V}}{\partial \phi} h \tau\right. \\
&\left.\quad+\frac{1}{4} e^{\phi / 2}\left(F^{M P} F_{P}^{N}-\frac{1}{4} F^{2} G^{M N}\right) \tau h_{M N}\right] \\
& S(V, \tau)=\int d^{D} X \sqrt{-G}\left[-\frac{1}{4} e^{\phi / 2} F_{M N}\left(D^{M} V^{N}-D^{N} V^{M}\right) \tau\right] \\
& S\left(V_{2}, V_{2}\right)=-\frac{1}{48} \int d^{D} X \sqrt{-G} e^{\phi} V_{[N P ; M]} V^{[N P ; M]}, \\
& S\left(V, V_{2}\right)=-\frac{\kappa}{12} \gamma \int d^{D} X \sqrt{-G} e^{\phi} V_{[N P ; M]} F^{[M N} V^{P]}
\end{aligned}
$$

where

$$
F_{[M N} V_{P]} \equiv F_{M N} V_{P}+2 \text { cyclic perms, } \quad V_{[N P ; M]} \equiv V_{N P ; M}+2 \text { cyclic perms. }
$$

We would like to remind the reader of the assumptions we have made to derive (3.6) and (3.9)-(3.15) (and (A.1)-(A.2) given in appendix A):

- If the Kalb-Ramond field and the term $H_{M N P} H^{M N P}$ in (2.3) is not included, then the only assumption we made is that the background satisfies the EOMs (2.7)-(2.10).

- If the Kalb-Ramond field and the term $H_{M N P} H^{M N P}$ in (2.3) is instead included, we also assumed $D=6$ and the background gauge field $\mathcal{A}$ to lie in the Cartan subalgebra.

We observe that if we want to focus on the $D$-dimensional EYM $\Lambda$ system we can restrict ourselves to the terms $S(h, h), S(V, V)$ (for $\gamma=0$ ), $S(h, V)$ and the $\xi$-dependent terms given in appendix A. Instead, if we want to consider the $6 \mathrm{D}$ supergravity, we should put $\gamma=1, \mathcal{V}(\phi)=8 g_{1}^{2} e^{-\phi / 2} / \kappa^{4}$ and also take into account the terms (3.11)-(3.15). Finally, we note that our results reduce to those of ref. [12] which studies a general non-supersymmetric class of thick brane models, once we take $T=0, \gamma=0$ and we neglect the fluctuations $V_{M N}$. 


\subsection{Local symmetries}

As a consequence of the local symmetries of the complete model, the linearized theory also possesses a number of local symmetries:

$$
\begin{aligned}
\delta h_{M N} & =-\eta_{N ; M}-\eta_{M ; N}, \\
\delta V_{M} & =-\eta^{L} F_{L M}-D_{M} \chi \\
\delta \tau & =-\eta^{M} \partial_{M} \phi \\
\delta V_{M N} & =2 \gamma \kappa \chi F_{M N}+\lambda_{N ; M}-\lambda_{M ; N}, \\
\delta \xi^{M} & =\eta^{M}(Y)-\zeta^{\alpha} \partial_{\alpha} Y^{M} .
\end{aligned}
$$

Eqs. (3.16), (3.17) and (3.18) represent the effect of the local symmetries (descending from the $D$-dimensional coordinate transformation invariance and gauge symmetry) on the metric, the gauge field and the dilaton fluctuations (see e.g. ref. [12]). The bulk functions $\eta$ and $\chi$ are the gauge functions associated with the $D$-dimensional coordinate invariance and gauge symmetry.

Eq. (3.19) represents instead a local symmetry acting on $V_{M N}$, which descends from both the gauge symmetry and the Kalb-Ramond symmetry. ${ }^{10}$ For this reason $\chi$ and $\lambda_{M}$ are independent (bulk) gauge functions. Let us explicitly check (3.19). To do so, it is enough to verify the invariance of the 3-form (3.4) under (3.17) and (3.19). We have

$$
\delta H^{(1)}=\frac{1}{\kappa} d\left(\delta V_{2}\right)+2 \gamma F \wedge \delta V=2 \gamma d(\chi F)+2 \gamma F \wedge(-\eta \cdot F-D \chi),
$$

where we have used $d^{2} \lambda=0$ and $\eta \cdot F$ represents the 1 -form with components $\eta^{M} F_{M N}$. Now, by using the $4 \mathrm{D}$ Poincare invariance of the background and $D=6$, which we always assume in the presence of the Kalb-Ramond field, we have $F \wedge(\eta \cdot F)=0$ and $F \wedge \mathcal{A}=0$; also, by remembering that $\mathcal{A}$ is assumed to lie in the Cartan subalgebra, we have $d F=0$. These equations are sufficient to conclude $\delta H^{(1)}=0$.

Finally, eq. (3.20) represents the local transformation of the perturbation of the brane position, descending from the $D$-dimensional coordinate invariance and the $4 \mathrm{D}$ brane coordinate transformation invariance (respectively the first and the second term on the right hand side of (3.20)); the latter invariance is associated to $\zeta^{\alpha}$ (a function of $x^{\alpha}$ ), which represents another independent gauge function.

\section{Perturbations in the light cone static gauge}

Having derived the general bilinear action, we now have to choose a gauge in order to study the physical spectrum. In this section we will discuss our gauge choice and give the corresponding bilinear action.

\footnotetext{
${ }^{10}$ By Kalb-Ramond symmetry we mean the local invariance under $B_{2} \rightarrow B_{2}+d \Lambda$ of the action, where $\Lambda$ is a general 1 -form.
} 


\subsection{Gauge fixing}

We have two types of local symmetries: the bulk local symmetries (which include the $D$-dimensional coordinate transformation invariance, the gauge symmetry and the KalbRamond symmetry) and the $4 \mathrm{D}$ coordinate transformation invariance on the brane. Let us start with the first group.

A very convenient gauge choice for the bulk local symmetry is the light cone gauge, as it ensures that the dynamics of sectors with different spin decouple at the bilinear level. ${ }^{11}$ Another advantage of the light cone gauge is that it does not involve gauge artifacts such as Faddeev-Popov ghosts, but contains only the physical spectrum [23-25]. To define this gauge, let us introduce $x^{( \pm)} \equiv\left(x^{3} \pm x^{0}\right) / \sqrt{2}$ and $A^{( \pm)} \equiv\left(A^{3} \pm A^{0}\right) / \sqrt{2}$, for a general vector $A^{M}$. Then the light cone gauge is defined by

$$
V_{(-)}=0, \quad h_{(-) M}=0, \quad V_{(-) M}=0, \forall M .
$$

It can be proved that, after imposing $(4.1)$, the $(+)$ components of the different fields (i.e. $V_{(+)}, h_{(+) M}$ and $V_{(+) M}$ ) are not independent, but can be expressed in terms of the other components by means of constraint equations [12, 24, 25]. We therefore end up with the following independent bulk fields: $h_{i j}, h_{i \underline{m}}, V_{i}, V_{i \underline{m}}, h_{\underline{m n}}, V_{\underline{m}}, V_{i j}, V_{\underline{m n}}$ and $\tau$, where $i, j, \ldots=1,2$. In particular the $h_{(++)}$field equation simply leads to the constraint

$$
h=0,
$$

which brings a considerable amount of simplification.

Concerning the $4 \mathrm{D}$ coordinate transformation invariance, we instead impose the condition $[15]$

$$
\xi^{\mu}=0 .
$$

We will refer to (4.3) as to the static gauge. We observe that the light cone gauge and the static gauge are compatible because, once we fix the light cone gauge by choosing $\eta_{M}, \chi$ and $\lambda_{M}$ in a suitable way, we still have the freedom to perform the local transformations generated by $\zeta^{\alpha}$. The static gauge is also free from Faddeev-Popov unphysical ghosts [15]. We observe that (4.3) does not remove completely the brane position fields $\xi^{M}$, but we are left with their components along the extra dimensions $\xi^{\underline{m}}$. We will refer to them as branons. Even if the branons represent physical degrees of freedom, it can happen that they can be consistently truncated e.g. by imposing an orbifold symmetry, as in the RS models or in the conical-GGP compactification [9]. In the following we will confirm that the spin- 0 fields $\xi^{\underline{m}}$ do not have any mixing with the spin- 2 and spin- 1 sectors in the light cone gauge.

\subsection{Bilinear action in the light cone static gauge}

Here we provide the bilinear action in the light cone static gauge, that we have computed by imposing the gauge conditions (4.1) and (4.3) on the general bilinear action and by using the constraint equations for the $(+)$ components. In this section we assume the form

\footnotetext{
${ }^{11}$ This has been observed in other studies, for example [12, 24-26].
} 
given in (2.12)-(2.17) for the background solution, and give the part of the action that is independent of the branons. Those involving the branons are given in appendix B.

The results that are presented here reduce to those for the non-supersymmetric model present in ${ }^{12}[12]$ once we take $T=0, \gamma=0$ and we neglect the fluctuations $V_{M N}$; they also correctly reduce (for $T=0$ and $\gamma=1$ ) to the results of [27], where the linear perturbations of the sphere-monopole solution to the 6D supersymmetric model are analyzed.

\subsubsection{Spin-2 action}

The spin-2 action $S^{(2)}$ only contains the field $\tilde{h}_{i j} \equiv h_{i j}-\frac{1}{2} G_{i j} h_{k}{ }^{k}$ and has the following simple expression in terms of $\tilde{h}_{i}^{j}=G^{j k} \tilde{h}_{i k}$ :

$$
S^{(2)}(h, h)=-\frac{1}{4 \kappa^{2}} \int d^{D} X \sqrt{-G} \partial_{M} \tilde{h}_{i}{ }^{j} \partial^{M} \tilde{h}_{j}{ }^{i} .
$$

We observe that (4.4) has exactly the same form as in [12] even if we have included the brane terms. Therefore, the brane sources do not explicitly contribute to the spin-2 dynamics. We shall use (4.4) to derive the $4 \mathrm{D}$ gravitational spectrum for the solutions described in Subsection 2.3.

\subsubsection{Spin-1 action}

The spin-1 action $S^{(1)}$ involves $h_{i \underline{m}}, V_{i}$ and $V_{i \underline{m}}$. We have the following explicit expressions.

$$
\begin{aligned}
S^{(1)}(h, h)=\int d^{D} X \sqrt{-G}[- & \frac{1}{2 \kappa^{2}}\left(\partial_{\mu} h_{i \underline{m}} \partial^{\mu} h^{i \underline{m}}+\partial_{\rho} h_{i \underline{m}} \partial_{\rho} h^{i \underline{m}}+h_{i \underline{m} ; n} h^{i \underline{m} ; n}\right) \\
& -\frac{1}{4 \kappa^{2}} h_{i m} h^{i m}\left(A^{\prime 2}+\frac{B^{\prime 2}}{2}\right)-\frac{1}{4 \kappa^{2}} h_{\rho i} h_{\rho}^{i}\left(D_{2} A^{\prime} B^{\prime}-A^{\prime 2}\right) \\
& -\frac{1}{2} h_{i \underline{\underline{m}}} h_{\underline{n}}^{i}\left(\frac{1}{2} e^{\phi / 2} F^{\underline{m}}{ }_{\underline{l}} F^{\underline{n l}}+\frac{1}{4 \kappa^{2}} \partial^{\underline{m}} \phi \partial^{\underline{n}} \phi\right) \\
& \left.+\frac{1}{\kappa^{2}} A^{\prime} h_{\rho}{ }^{i} h_{m i} ; m-\frac{T}{4} \sqrt{g / G} \delta\left(X_{c}-Y_{c}\right) h_{\underline{m} i} h^{\underline{m}} i\right]
\end{aligned}
$$

where $^{\prime} \equiv \partial_{\rho}$. The last term in (4.5) is the brane contribution. We have introduced the notation $X_{c}$ and $Y_{c}$ for the internal components of the coordinate and the brane position respectively, where the label $c$ stands for the codimension of the brane. The other non

\footnotetext{
${ }^{12}$ We do, however, correct some typos in that reference.
} 
vanishing terms are the following.

$$
\begin{aligned}
& S^{(1)}(V, V)=\int d^{D} X \sqrt{-G} e^{\phi / 2}\left[-\frac{1}{2}\left(\partial_{\mu} V_{i} \partial^{\mu} V^{i}+e^{-A} \partial_{\rho} V_{i} \partial_{\rho} V_{i}+D_{m} V_{i} D^{m} V^{i}\right)\right. \\
& \left.-\frac{\kappa^{2}}{4} \gamma^{2} e^{\phi / 2}\left(F_{\underline{m n}} V_{i}\right) F \underline{m n} V^{i}\right] \text {, } \\
& S^{(1)}(h, V)=\int d^{D} X \sqrt{-G} e^{\phi / 2}\left(-D_{\underline{m}} V_{i} h_{\underline{l}}^{i} F \frac{l m}{2}-\frac{1}{2} A^{\prime} V_{i} h^{\underline{l i}} F_{\underline{l} \rho}\right), \\
& S^{(1)}\left(V_{2}, V_{2}\right)=-\frac{1}{8} \int d^{D} X \sqrt{-G} e^{\phi}\left\{e ^ { - A } \left[\partial_{\mu} V_{i \underline{m}} \partial^{\mu} V_{i} \underline{m}\right.\right. \\
& \left.+G \frac{m l}{\underline{m} \underline{n h}}\left(\partial_{\underline{m}} V_{\underline{n} i} \partial_{\underline{l}} V_{\underline{h} i}-\partial_{\underline{m}} V_{\underline{n} i} \partial_{\underline{h}} V_{\underline{l i}}\right)\right] \\
& -e^{-4 A-2 \phi}\left(e^{\phi+3 A / 2} V^{\underline{m}}\right)_{; \underline{m}}\left(e^{\phi+3 A / 2} V^{\underline{n}}{ }_{i ; \underline{n}}\right. \\
& -2 e^{-2 A} V_{\underline{m} i} \partial^{\underline{m}}\left[e^{-\phi-A / 2}\left(e^{\phi+3 A / 2} V^{\underline{n}}{ }_{i, \underline{n}}\right]\right\} \text {, } \\
& S^{(1)}\left(V, V_{2}\right)=-\frac{\kappa}{2} \gamma \int d^{D} X \sqrt{-G} e^{\phi}\left(-\frac{1}{2} A^{\prime} V_{i m} V^{i} F_{\rho}{ }^{m}+V_{\underline{n} i ; \underline{m}} F^{\underline{m n}} V^{i}\right) .
\end{aligned}
$$

The term $S^{(1)}\left(h, V_{2}\right)$ vanishes as a consequence of $H_{M N P}=0$ (at the background level). We observe that the term $S^{(1)}(V, V)$ reduces, as it should, to the corresponding action in ref. [6] in the case in which $V_{i}$ is orthogonal to the background gauge field. Finally, we note that the brane tension enters explicitly only in the term $S^{(1)}(h, h)$.

\subsubsection{Spin-0 action and singularities due to backreacting, fluctuating branes}

The last and most complicated part is the spin-0 action, which involves ${ }^{13} h_{\underline{m n}}, h_{i}{ }^{i}, V_{\underline{m}}$, $\tau, V_{i j}, V_{\underline{m n}}$ and $\xi^{\underline{m}}$. We observe that, in the light cone static gauge, the fields $\xi^{\underline{m}}$ indeed only appear here. In other words they are in general completely decoupled from the spin-2 and spin-1 fields. Since it is quite complicated, we give the explicit expression of the spin-0 action in appendix B.

Having completed the bilinear action, we should make some observations regarding its consistency, in particular given the presence of infinitesimally thin dynamical sources. Indeed, as to be expected, if we include the gravitational backreaction of the branes $(T \nrightarrow$ 0 ) then there are singular contributions to the dynamics of both the bulk gravitational fluctuations and the branons.

First, concerning the bulk gravitational fluctuations, we encounter localized contributions to the mass terms in both the spin-1 (see eq. (4.5)) and spin-0 (see eq. (B.1)) sectors. These contributions involve the behaviour of background and perturbed fields at the background positions of the branes. They are well-defined in the codimension one RS scenario, where the metric is well-defined everywhere including at the brane positions (although its derivatives are not). They do not appear to be well-defined in the codimension two (or higher) case, where the internal metric is actually singular at the brane positions due to the

\footnotetext{
${ }^{13}$ Note that $h_{\underline{m n}}$ and $h_{i}{ }^{i}$ are not independent as eq. (4.2) implies $h_{i}{ }^{i}+h_{\underline{m}} \underline{m}=0$.
} 
backreaction of the branes. However, as we shall see in subsequent sections, these terms do not obstruct our derivation of the 4D particle spectra arising from bulk modes in 6D.

Meanwhile, the linearized dynamics for the branons of a backreacting brane would be more problematic. For example, in (B.13), since the action is evaluated at the background position of the branes, the kinetic term for the branons is not well-defined in the codimension two case, because of the conical defect in $G_{m n}$. Such a singularity was discussed in [13], where it was argued that within the domain of validity of the effective field theory, the curvature singularity could be discarded. Moreover, the mass term for the branons takes the form ${ }^{14}$ of a $\delta(0)$. These singularities are not present in the RS model, as there the branons are projected out with an orbifolding. ${ }^{15}$ Indeed we should reiterate here that we apply our analysis to codimension one branes only on orbifold fixed points (to avoid the appearance of Gibbons-Hawking boundary terms), and so without branon degrees of freedom.

In order to perform a complete analysis of the spin- 0 action in codimension two (or higher) models, taking into account both the backreaction of the brane and its dynamical fluctuations, it seems necessary to resolve the thin structure of the brane. Otherwise we can assume a brane tension much smaller than the $6 \mathrm{D}$ fundamental scale, so that its backreaction is negligible. Or we can assume a high brane tension so that the brane is very heavy and rigid and does not oscillate. Or else we can assume an additional orbifold symmetry under which the branons are projected out - an example of such a symmetry has been provided in ref. [9] and is discussed in $^{16}$ appendix $\mathrm{C}$. In these cases, we can avoid the singular branon action.

\section{$5 \quad 6 \mathrm{D}$ (and 5D) braneworlds}

In the second part of this paper, we apply the results of the previous sections to derive the $4 \mathrm{D}$ particle spectra in specific setups. Our main interest is in the warped (and unwarped) axi-symmetric braneworld compactifications of $6 \mathrm{D}$ supergravity, but along the way we shall also discuss the rugbyball compactifications in the non-supersymmetric 6D EYM $\Lambda$ theory, as well as the 5D Randall-Sundrum models. We discuss in order the spin-2, spin-1 and spin-0 fluctuations.

\subsection{Gravitational fluctuations}

The simplest application of our results is the analysis of the spin-2 sector. As we have discussed, this sector completely decouples from the rest. The $\tilde{h}_{i}^{j}$ fields contain only the maximal helicity components of a spin-2 multipet; one should look for the remaining

\footnotetext{
${ }^{14} \delta(0)$ singularities due to the localization of fields on a boundary have been discussed in a different context (5D SYM theory on $\left.S^{1} / Z_{2}\right)$ in [28].

${ }^{15}$ Indeed, in the RS literature, the radion has been studied in depth [29], but the branons are absent. Although the radion can also be seen as a brane bending in the case of RS, since the branes are at the boundaries of the internal space, one should not confuse the radion with the branon. The radion is a deformation of the bulk metric, whereas the branon is a deformation of the brane itself within the bulk manifold. As a check of our formalism, we will find the radion mode in Subsection 5.4.

${ }^{16}$ By using the explicit expression for the mixing terms between branons and bulk fields given in appendix $\mathrm{B}$, it is easy to confirm that symmetry consistently truncates the branons.
} 
components in the spin- 1 and spin- 0 actions. However, by virtue of $4 \mathrm{D}$ Poincaré invariance, the lower helicity components must have the same spectrum [12]. We can therefore focus on eq. (4.4) to study the spin-2 fluctuations.

In order to analyze this sector we deduce the EOMs from eq. (4.4):

$$
\partial_{M}\left(\sqrt{-G} \partial^{M} \tilde{h}_{i}^{j}\right)=0 \quad \forall i, j .
$$

In deriving this equation we have required as usual that the boundary terms which emerge in the integration by parts vanish, that is

$$
\int d^{D} X \partial_{M}\left(\sqrt{-G} \delta \tilde{h}_{i}^{j} \partial^{M} \tilde{h}_{i}^{j}\right)=0
$$

where $\delta \tilde{h}_{i}^{j}$ is the variation of the field $\tilde{h}_{i}^{j}$, which is performed to apply the minimal action principle. Since we assume standard boundary conditions on the 4D boundary, (5.2) reduces to $[6,30]$

$$
\int d^{D_{2}+1} X \partial_{\underline{m}}\left(\sqrt{-G} \delta \tilde{h}_{i}^{j} \partial^{\underline{m}} \tilde{h}_{i}^{j}\right)=0 .
$$

We now perform a KK decomposition of the fields as follows:

$$
\tilde{h}_{i}^{j}(X)=\sum_{\mathbf{k}} \tilde{h}_{i}^{(\mathbf{k}) j}(x) f_{\mathbf{k}}(\rho, y)
$$

where $\mathbf{k}$ represents a collective KK number. By taking $\tilde{h}_{i}^{(\mathbf{k}) j}(x)$ to be an eigenfunction of $\eta^{\mu \nu} \partial_{\mu} \partial_{\nu}$, that is $\eta^{\mu \nu} \partial_{\mu} \partial_{\nu} \tilde{h}_{i}^{(\mathbf{k}) j}(x)=M_{\mathbf{k}}^{2} \tilde{h}_{i}^{(\mathbf{k}) j}(x)$, the EOMs (5.1) become

$$
-\frac{1}{\sqrt{-G}} e^{A} \partial_{\underline{m}}\left(\sqrt{-G} \partial^{\underline{m}} f_{\mathbf{k}}\right)=M_{\mathbf{k}}^{2} f_{\mathbf{k}}
$$

and the corresponding boundary conditions (5.3) read (we recall that $\delta \tilde{h}_{i}^{j}$ and $\tilde{h}_{i}{ }^{j}$ are independent fields)

$$
\int d^{D_{2}+1} X \partial_{\underline{m}}\left(\sqrt{-G} f_{\mathbf{k}^{\prime}} \partial^{\underline{m}} f_{\mathbf{k}}\right)=0 . \quad \forall \mathbf{k}, \mathbf{k}^{\prime}
$$

Condition (5.6) ensures that the operator acting on $f_{\mathbf{k}}$ in the left hand side of (5.5) is a Hermitian operator [6,30]; we will therefore refer to (5.6) as the hermiticity condition $(\mathrm{HC})$. In addition to the $\mathrm{HC}$ we will also require the wave functions $f_{\mathbf{k}}$ to be normalizable, that is

$$
\int d^{D_{2}+1} X \sqrt{-G} e^{-A} f_{\mathbf{k}}^{2}<\infty
$$

This normalizability condition (NC) is equivalent to the finiteness of the kinetic energy of the modes $\tilde{h}_{i}^{(\mathbf{k}) j}(x)$. We observe that there is always a constant massless $\left(M_{\mathbf{k}}^{2}=0\right)$ solution to (5.5), satisfying the $\mathrm{HC}$ (5.6). This solution corresponds to a $4 \mathrm{D}$ graviton provided that the NC is satisfied, that is $\int d^{D_{2}+1} X \sqrt{-G} e^{-A}<\infty$. 


\subsubsection{Randall-Sundrum}

In the special case $D=5$, and therefore in particular for the RS background (2.18), the EOM (5.5) has the form

$$
-e^{-A} \partial_{\rho}\left(e^{2 A} \partial_{\rho} f_{\mathbf{k}}\right)=M_{\mathbf{k}}^{2} f_{\mathbf{k}} .
$$

Here we do not want to analyze the latter equation as this has been done in the original RS works, but we observe, as a check of our spin-2 action, that (5.7) has exactly the same form as in [2].

\subsubsection{D brane worlds}

We now move to the conical-GGP solutions to 6D supergravity given in eqs. (2.21)-(2.22). Since our internal space is topologically $S^{2}$, we require $\tilde{h}_{i}^{j}$ to be periodic functions of $\varphi$ :

$$
\tilde{h}_{i}^{j}(X)=\sum_{\mathbf{n}, \mathbf{m}} \tilde{h}_{i \mathbf{n m}}^{j}(x) f_{\mathbf{n m}}(\rho) e^{i \mathbf{m} \varphi}
$$

where $\mathbf{m}$ is a generic integer and $\mathbf{n}$ is an extra KK number that emerges as we have a number of compact dimensions greater than one. Also we observe that eq. (5.5) with the $\mathrm{HC}$ and $\mathrm{NC}$ is formally identical ${ }^{17}$ to the corresponding problem for $4 \mathrm{D}$ gauge fields addressed in ref. [6]. Therefore, here we only give the result. The wave functions can be expressed in a more compact way by introducing

$$
\psi \equiv e^{(3 A+B) / 4} f
$$

where we have suppressed $\mathbf{n}$ and $\mathbf{m}$. The explicit expression for $\psi$ is

$$
\psi \propto z^{\epsilon}(1-z)^{\beta} F(a, b, c, z)
$$

where $z \equiv \cos ^{2}\left(u / r_{0}\right), F$ is Gauss's hypergeometric function and

$$
\begin{aligned}
\epsilon & \equiv \frac{1}{4}(1+2|\mathbf{m}| \bar{\omega}), \quad \beta \equiv \frac{1}{4}(1+2 \mathbf{m} \omega), \quad c \equiv 1+|\mathbf{m}| \bar{\omega}, \\
a & \equiv \frac{1}{2}+\frac{\mathbf{m}}{2} \omega+\frac{|\mathbf{m}|}{2} \bar{\omega}+\frac{1}{2} \sqrt{r_{0}^{2} M^{2}+1+\mathbf{m}^{2}(\omega-\bar{\omega})^{2}} \\
b & \equiv \frac{1}{2}+\frac{\mathbf{m}}{2} \omega+\frac{|\mathbf{m}|}{2} \bar{\omega}-\frac{1}{2} \sqrt{r_{0}^{2} M^{2}+1+\mathbf{m}^{2}(\omega-\bar{\omega})^{2}},
\end{aligned}
$$

with

$$
\omega \equiv(1-\delta / 2 \pi)^{-1}, \quad \bar{\omega} \equiv(1-\bar{\delta} / 2 \pi)^{-1} .
$$

Moreover the explicit form of the mass spectrum is given by

$$
M^{2}=\frac{4}{r_{0}^{2}}\left[\mathbf{n}(\mathbf{n}+1)+\left(\frac{1}{2}+\mathbf{n}\right)|\mathbf{m}|(\omega+\bar{\omega})+\mathbf{m}^{2} \omega \bar{\omega}\right] \geq 0
$$

\footnotetext{
${ }^{17}$ In [6] there is the extra parameter $N_{V}$, which is equal to zero here. To check that the two problems are identical it is useful to remember $A=\phi / 2$, which is true for the conical-GGP solutions. Also, take care that $\phi$ in reference [6] is half $\phi$ here.
} 

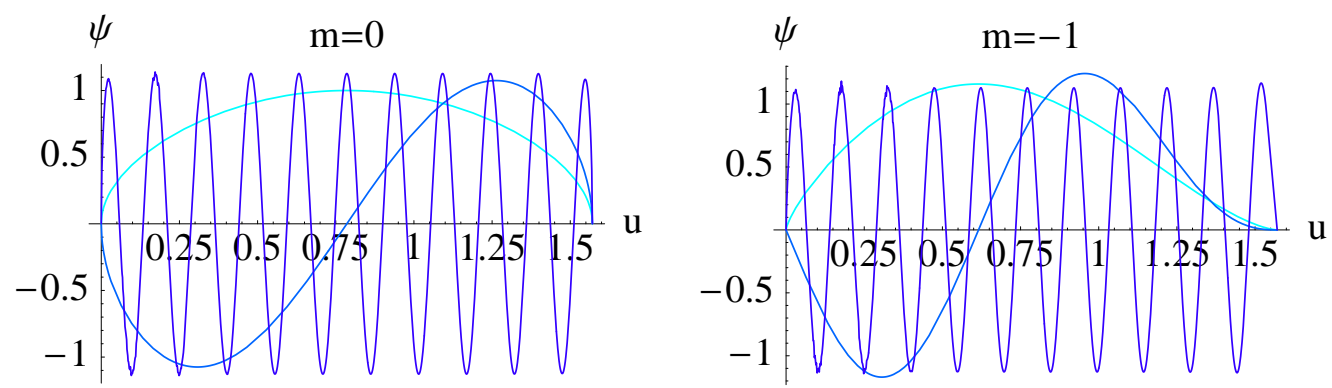

Figure 1. Graviton Wave Function Profiles: $\mathbf{n}=0,1,20$ modes plotted for angular momentum numbers $\mathbf{m}=-1,0$. The parameters are chosen to be $\left(r_{0}, \omega, \bar{\omega}\right)=(1,1 / 4,1)$, corresponding to a single negative tension brane at $u=0$. Also the normalization constant is set such that $\int d u|\psi|^{2}=1$. The number of intersections with the $\mathrm{u}$-axis equals $n$, according to quantum mechanics. Notice that the $(\mathbf{m}, \mathbf{n})=(0,0)$ mode is massless.

where $\mathbf{n}=0,1,2,3, \ldots[6]$. So we have obtained the exact and complete spectrum (wave functions and masses) for the spin-2 fluctuations of the conical-GGP solutions. We observe that eq. (5.13) tells us there is a massless normalizable solution (for $\mathbf{n}=\mathbf{m}=0$ ), which corresponds to the $4 \mathrm{D}$ graviton. This solution is separated from the first KK excitation by a finite mass gap, which is of order $1 / r_{0}$ (if $\omega \sim \bar{\omega} \sim 1$ ). We plot some representative wave function profiles in figure 1. As discussed in [6] the asymptotic behaviour close to the branes is universal for each KK tower, and it does not appear possible to separate the infinite number of heavy modes from the light ones by using their respective wave function profiles.

Here we also observe that eq. (5.1) is independent of $\gamma$ and the dilaton potential $\mathcal{V}$. This implies that the spin-2 spectrum of the non supersymmetric and supersymmetric models are the same (provided the backgrounds are the same). Indeed, the rugbyball configuration (that is $\omega=\bar{\omega}$ ) leads to the same spin-2 spectrum in the EYM $\Lambda$ model and in the $6 \mathrm{D}$ supergravity.

Finally, as a check, we can consider the $S^{2}$ limit $(\omega, \bar{\omega} \rightarrow 1)$, whose mass spectrum is well-known. Our spectrum (5.13) reduces to

$$
\frac{r_{0}^{2}}{4} M^{2}=l(l+1), \quad \text { multiplicity }=2 l+1,
$$

where $l=0,1,2,3, \ldots$. Since $r_{0} / 2$ represents the radius of $S^{2}$ in the sphere limit, this is exactly the result that one finds by using the spherical harmonic expansion [31, 32] from the beginning.

\subsection{Vector fluctuations}

Here we analyze the vector fluctuations, in particular their wavefunction expansions and mass spectra. In the following subsection we shall study the implications of these results for the structure of the 4D gauge group in the $6 \mathrm{D}$ models of interest. The physical 4D vector field spectrum can be extracted from the spin-1 action given in Subsection 4.2.2. 
However, some of the perturbations in that action are simply the helicity- $( \pm 1)$ components of massive gravitons and therefore should not be interpreted as independent vector fields.

\subsubsection{Randall-Sundrum}

To illustrate the previous point we first notice that our spin-1 action leads to the well-known result that there are no physical $4 \mathrm{D}$ vector fields in the RS model (unless one introduces bulk gauge fields). Indeed, in that case the only field appearing in the spin-1 action is $h_{i \rho}$, whose action is simply

$$
S^{(1)}(h, h) \propto \int d^{5} X \sqrt{-G}\left(\partial_{\mu} h_{i \rho} \partial^{\mu} h_{\rho}^{i}+\partial_{\rho} h_{i \rho} \partial_{\rho} h_{\rho}^{i}-\frac{A^{\prime 2}}{2} h_{i \rho} h_{\rho}^{i}-\frac{3}{2} A^{\prime \prime} h_{i \rho} h_{\rho}^{i}\right),
$$

where we used the property

$$
A^{\prime \prime}+T \kappa^{2} \delta(\rho-Y) / 3=0
$$

that follows from the form of the warp factor, eq. (2.18), in the RS model. Therefore, once (5.16) is used the problem assumes the same form as in ref. [12], where it is shown that the $4 \mathrm{D}$ spectrum from $h_{i \rho}$ exactly reproduces the graviton one with the zero mode removed. By counting the degrees of freedom, it follows that there are no physical vector fields.

\subsubsection{D brane worlds}

Let us begin by considering what we might expect from the symmetries of the problem with some benefit of hindsight from the authors. In the limit where the brane tensions go to zero, the smooth sphere-monopole compactification is recovered. In this case, standard KK theory tells us that there are three massless KK gauge bosons, ${ }^{18}$ which manifest the $\mathrm{SU}(2)$ isometries of the sphere in the $4 \mathrm{D}$ theory [31]. Clearly, any branes break the spherical symmetry in the internal dimensions. For the solutions of present interest an axial isometry survives, and therefore we can expect the $4 \mathrm{D}$ theory to enjoy a U(1) KK gauge symmetry. ${ }^{19}$

At the same time, for the case of an unwarped "saddle-sphere" with the special deficit angles (for $\alpha=1$ we recover the sphere)

$$
\delta=-2 \pi,-4 \pi, \ldots \quad \text { or } \quad \alpha=\frac{1}{\omega}=2,3, \ldots
$$

the metric (2.27) - defined everywhere but at the branes - has three single-valued Killing vectors, which obey the Lie algebra of $\mathrm{SU}(2)$ :

$$
K^{+}=e^{i \alpha \varphi}\left(\frac{\partial}{\partial \theta}+i \cot \theta \frac{1}{\alpha} \frac{\partial}{\partial \varphi}\right), K^{-}=e^{-i \alpha \varphi}\left(-\frac{\partial}{\partial \theta}+i \cot \theta \frac{1}{\alpha} \frac{\partial}{\partial \varphi}\right), K^{0}=-\frac{i}{\alpha} \frac{\partial}{\partial \varphi} .
$$

\footnotetext{
${ }^{18}$ There may be additional massless gauge fields descending from any unbroken higher dimensional gauge symmetries.

${ }^{19}$ For the analysis of a very similar model, in which the $\mathrm{SU}(2) \mathrm{KK}$ gauge symmetry of the sphere is broken down to $\mathrm{U}(1)$ by smooth axisymmetric deformations, see ref. [33].
} 
Only one of these Killing vectors, $K^{0}$, implies a genuine continuous isometry, since $K^{ \pm}$ cannot be globally integrated to an isometry. ${ }^{20}$ In other words, we have an infinitesimal $\mathrm{SU}(2)$ isometry for the special saddle-spheres, compared to a genuine $\mathrm{SU}(2)$ isometry for the sphere. As we will show, this turns out to be sufficient to ensure three massless 4D vectors amongst the KK spectra. From the point of view of the full $4 \mathrm{D}$ theory, however, we will argue that these massless fields arise accidentally and that their masslessness is not protected by any symmetry.

Rugbyball harmonics Let us now see how the above story plays out in detail. Our focus shall then be on the unwarped rugbyballs and saddle-spheres, eq. (2.27), and indeed all previous results have indicated that warping does not lead to any qualitative changes in the physics (see the spin-2 results in the present paper, as well as refs. [6]-[11]). We shall thus proceed by finding a set of "rugbyball harmonics" and their mass spectra, in analogy to the spherical harmonics (and, more generally, the so-called Wigner functions) used in the smooth sphere compactification [31].

We first observe that the vector field fluctuations $V_{i}$, which are orthogonal to the gauge field background $\left(V_{i} F_{\underline{m n}}=0\right)$, do not mix with the other perturbations $h_{i \underline{m}}$ and $V_{i \underline{m}}$. These fluctuations have been already studied in ref. [6] where the complete KK towers are provided and it is shown that there are as many $4 \mathrm{D}$ gauge fields as fluctuations $V_{i}$ with vanishing monopole charge $\left(V_{i} \times F_{\underline{m n}}=0\right)$, as expected from group theory. Here we therefore consider only the case when $V_{i}$ is parallel to the background monopole.

Now, from Subsection 4.2.2 it follows that the spin-1 action for unwarped solutions has the following form.

$$
\begin{aligned}
S^{(1)}(h, h) & =-\frac{1}{2 \kappa^{2}} \int d^{6} X \sqrt{-G}\left(\partial_{\mu} h_{m i} \partial^{\mu} h^{m i}+h_{m i ; n} h^{m i ; n}+\frac{R}{2} h_{m i} h^{m i}\right), \\
S^{(1)}(V, V) & =-\frac{1}{2} \int d^{6} X \sqrt{-G}\left[\partial_{\mu} V_{i} \partial^{\mu} V^{i}+\partial_{m} V_{i} \partial^{m} V^{i}+\frac{\kappa^{2}}{2} \gamma^{2} F^{2} V_{i} V^{i}\right], \\
S^{(1)}\left(V_{2}, V_{2}\right) & =-\frac{1}{8} \int d^{6} X \sqrt{-G}\left(\partial_{\mu} V_{m i} \partial^{\mu} V^{m i}+V_{m i ; n} V^{m i ; n}+\frac{R}{2} V_{m i} V^{m i}\right), \\
S^{(1)}(h, V)+S^{(1)}\left(V, V_{2}\right) & =\int d^{6} X \sqrt{-G}\left(-\partial_{m} V_{i} h_{n i} F^{n m}-\frac{\kappa}{2} \gamma \partial_{m} V^{i} V_{n i} F^{n m}\right),
\end{aligned}
$$

where we have used $V_{i ; m}=\partial_{m} V_{i}$ because the background solution is unwarped and $V_{i}$ is uncharged under the background monopole. Also $m, n, \ldots$ here run over $\theta$ and $\varphi$. To derive the last term in $S^{(1)}(h, h)$ we have used the Einstein equations:

$$
\frac{2}{\kappa^{2}} \sqrt{-G} R_{m n}=\sqrt{-G} F_{m l} F_{n}{ }^{l}+G_{m n} T \delta\left(X_{2}-Y_{2}\right),
$$

which allow us to rewrite the brane contribution in the last term of (4.5) as a combination of the Ricci tensor and the field strength. Also we have used that in two dimensions

\footnotetext{
${ }^{20}$ To avoid the need of differential geometric results for singular spaces, we can consider removing the brane singularities and taking instead a smooth non-compact manifold, for which $0<\theta<\pi$. Killing vector fields are the generators of the infinitesimal isometries of a manifold, whereas an isometry is a global aspect of the geometry. Whilst for smooth compact manifolds the Killing vectors are always globally integrable to an isometry, for non-compact manifolds this may not always be the case.
} 
$R_{m n}=G_{m n} R / 2$ and the Maxwell equations $F_{; m}^{m n}=0$. The EOMs descending from (5.19) are the following.

$$
\begin{aligned}
\left(\partial^{2}+D^{2}-\frac{R}{2}\right) h_{m i}-\kappa^{2} F_{m}{ }^{l} \partial_{l} V_{i} & =0, \\
\left(\partial^{2}+D^{2}-\frac{R}{2}\right) V_{m i}-2 \kappa \gamma F_{m}{ }^{l} \partial_{l} V_{i} & =0, \\
\left(\partial^{2}+D^{2}-\frac{\kappa^{2}}{2} \gamma^{2} F^{2}\right) V_{i}+F^{n m} h_{n i ; m}+\frac{\kappa}{2} \gamma F^{n m} V_{n i ; m} & =0,
\end{aligned}
$$

where $\partial^{2} \equiv \partial_{\mu} \partial^{\mu}$ and $D^{2} \equiv D_{m} D^{m}$. As for the spin-2 case above, the EOMs come with a set of boundary conditions, which we refer to as Hermiticity Conditions (HCs) [6, 30]:

$$
\begin{aligned}
\int d^{6} X \sqrt{-G}\left(\delta h_{m i} h^{m i ; n}\right)_{; n} & =0, \quad \int d^{6} X \sqrt{-G}\left(\delta V_{m i} V^{m i ; n}\right)_{; n}=0, \\
\int d^{6} X \sqrt{-G}\left(\delta V^{i} h_{l i} F^{l m}\right)_{; m} & =0, \quad \int d^{6} X \sqrt{-G}\left(\delta V^{i} V_{l i} F^{l m}\right)_{; m}=0, \\
\int d^{6} X \sqrt{-G}\left(\delta V_{i} \partial^{m} V^{i}\right)_{; m} & =0 .
\end{aligned}
$$

We will additionally impose the usual Normalizability Conditions (NCs).

We can immediately observe that there is a simple solution to eqs. (5.21)-(5.23), with $h_{\theta i}=h_{\varphi i}=0, V_{\theta i}=V_{\varphi i}=0$ and $V_{i}$ independent of the extra dimensions. Its squared mass is given by

$$
M^{2}=\frac{\kappa^{2}}{2} \gamma^{2} F^{2}=\frac{8}{r_{0}^{2}} \gamma^{2}
$$

where we explicitly used the rugbyball solution. We see that the monopole $\mathrm{U}(1)$ is a gauge symmetry in the EYM $\Lambda$ model $(\gamma=0)$, whereas it is broken in $6 \mathrm{D}$ supergravity $(\gamma=1)$, like for the smooth sphere-monopole solution [16].

We now want to find the general solution to eqs. (5.21)-(5.23) subject to the HCs and NCs. System (5.21)-(5.23) is a rather complicated set of coupled differential equations, but the case at hand can be elegantly solved by using the harmonic expansion of the scalar Laplacian; let us now describe this technique. It is easy to solve the eigenvalue problem for $-D^{2}$ acting on the $2 \mathrm{D}$ scalars (in fact, for $A=0$ and applying the diagonal $\mathrm{HC}$ and the NC, the system is identical to that for the helicity-2 field above). The eigenfunctions are then given by (5.8)-(5.11) with $\omega=\bar{\omega}$, and the eigenvalues can be written as:

$$
\mu_{\mathbf{n} \mathbf{m}}^{2} \equiv \frac{4}{r_{0}^{2}}(\mathbf{n}+|\mathbf{m}| \omega)(\mathbf{n}+|\mathbf{m}| \omega+1) \geq 0
$$

where $\mathbf{n}=0,1,2,3, \ldots$ and $\mathbf{m}$ run over all the integers. This is the generalization to the rugbyball of the scalar spherical harmonics. They form a complete basis for the $2 \mathrm{D}$ scalar fields $V_{i}$.

We next proceed by determining a complete basis for the 2D vector fluctuations $\left\{h_{\theta i}, h_{\varphi i}\right\}$ and $\left\{V_{\theta i}, V_{\varphi i}\right\}$. We focus only on $\left\{h_{\theta i}, h_{\varphi i}\right\}$ as the analysis for $\left\{V_{\theta i}, V_{\varphi i}\right\}$ is identical. A way to determine such a basis is to look at the eigenvalue problem for the operator $-D^{2}+R / 2$ appearing in eq. (5.21), because the diagonal HC (5.24) guarantees that 
this operator is Hermitian over the space of functions where $\left\{h_{\theta i}, h_{\varphi i}\right\}$ lives, and therefore has a complete basis of eigenfunctions. Again, this system is easy to solve, this time using the results ${ }^{21}$ of ref. [9]. We therefore just summarise the results. The eigenvalue problem for $-D^{2}+R / 2$ on $2 \mathrm{D}$ vectors generically mixes the $h_{\theta i}$ and $h_{\varphi i}$ components, but reduces to a diagonal form, at least in the rugbyball case, by introducing ${ }^{22}$

$$
h_{ \pm i} \equiv \frac{1}{\sqrt{r_{0}}}\left(e^{B / 4} h_{\theta i} \pm i e^{-B / 4} h_{\varphi i}\right) .
$$

Eq. (5.29) defines a new basis for tensors on the 2D internal space, and we remind the reader that for the rugbyball $e^{B}=\alpha^{2} \sin ^{2} \theta$. The squared mass problem can then be transformed into a pair of decoupled Schrödinger equations, which can be solved. Note that the singularities of the spin-1 action discussed in Subsection 4.2.3 appear in the Schrödinger problems as two singular points in the effective potentials (one for each brane), which do not obstruct the determination of the spectrum $[6,9]$. The $h_{ \pm i}$ fields can be KK expanded as follows

$$
h_{ \pm i}(X)=\sum_{\mathbf{n}, \mathbf{m}} h_{ \pm i \mathbf{n} \mathbf{m}}(x) f_{\mathbf{n} \mathbf{m}}^{ \pm}(\theta) e^{i \mathbf{m} \varphi}
$$

and, in the case

$$
\mathbf{m}=0 \quad \text { or } \quad|\mathbf{m}| \geq 1 / \omega,
$$

both the KK tower associated with $f^{+}$and $f^{-}$turn out to be exactly that in (5.28), where $\mathbf{n}=0,1,2,3, \ldots$ and $\mathbf{m}$ run over all the integers, but with the constraint $\{\mathbf{n}, \mathbf{m}\} \neq\{0,0\}$. Condition (5.31) is satisfied by every $|\mathbf{m}|$ for non-negative tensions and it is satisfied by some (but not all) $|\mathbf{m}|$ for negative tensions. This, however, will be enough to show that when the tensions assume the values in (5.17), the KK spectra include extra massless spin-1 fields. In the following we denote (5.31) with $0 \nless|\mathbf{m}| \omega \nless 1$.

So we have found that, for modes satisfying (5.31), the spectrum of $-D^{2}+R / 2$ on $2 \mathrm{D}$ vectors is made up of two identical copies of the spectrum of $-D^{2}$ on $2 \mathrm{D}$ scalars but with zero mode removed. This suggests that we may be able to express the eigenfunctions of $-D^{2}+R / 2$ on $2 \mathrm{D}$ vectors in terms of eigenfunctions of $-D^{2}$ on $2 \mathrm{D}$ scalars. Indeed, if we consider a solution $V$ to the eigenvalue problem of $-D^{2}$ with eigenvalue $\mu^{2}$, then it is easy to show that $\partial_{m} V$ is an eigenfunction of $-D^{2}+R / 2$ with the same eigenvalue. In the case (5.31), this implies that we can write

$$
\partial_{ \pm}\left(f_{\mathbf{n} \mathbf{m}}(\theta) e^{i \mathbf{m} \varphi}\right)=c_{\mathbf{n} \mathbf{m}} f_{\mathbf{n} \mathbf{m}}^{ \pm}(\theta) e^{i \mathbf{m} \varphi}
$$

where we have used the basis defined in eq. (5.29) for $\partial_{ \pm}$, and moreover $c_{\mathbf{n} \mathbf{m}}$ are normalization constants which, having chosen a convenient normalization for the wave functions, can be fixed to be $c_{\mathbf{n} \mathbf{m}}=\mu_{\mathbf{n} \mathbf{m}} / \sqrt{2}$. This is the analogue of the derivative relation that exists between Wigner functions for fields of different spin on the sphere (see eq. (3.17) of

\footnotetext{
${ }^{21}$ In ref. [9] a more general problem has been solved, which reduces to the present one in the unwarped case $A=0$.

${ }^{22}$ The \pm appearing in (5.29) and throughout this section should not be confused with the $( \pm)$ used to defined the light-cone gauge in Subsection 4.1, for this reason the latter are written inside brackets.
} 
ref. [31]). So, remarkably, we can construct the complete harmonic expansion for ${ }^{23} V_{i}, h_{ \pm i}$ and $V_{ \pm i}$ by using the solution to the eigenvalue problem for the scalar Laplacian. Moreover, it is easy to check that, having applied the diagonal HCs to derive the complete basis for $h_{ \pm i}$ and $V_{ \pm i}$, the mixed HCs (5.25) are automatically satisfied.

Having derived the harmonic expansions one more observation is necessary. It turns out that the factor $F_{+}{ }^{+}$which appears in the mixing terms $S^{(1)}(h, V)$ and $S^{(1)}\left(V_{2}, V\right)$ is constant for the rugbyball $\left(F_{+}^{+}=2 \sqrt{2} i /\left(r_{0} \kappa\right)\right)$. Putting everything together, we are then able to transform the differential eigenvalue problem for the squared mass operator into an algebraic problem that can be solved. In particular, after integrating out the extra dimensions, Action (5.19) assumes the following form in terms of the KK modes.

$$
\begin{aligned}
& S^{(1)}(h, h)+S^{(1)}\left(V_{2}, V_{2}\right)+S^{(1)}(h, V)+S^{(1)}\left(V, V_{2}\right)+S^{(1)}(V, V) \\
& =\int d^{4} x \sum_{\mathbf{n}, \mathbf{m}}\left\{\frac{1}{2 \kappa^{2}}\left(h_{+i \mathbf{n} \mathbf{m}}\right)^{*}\left(\partial^{2}-\mu_{\mathbf{n} \mathbf{m}}^{2}\right) h_{+i \mathbf{n} \mathbf{m}}+\frac{1}{2 \kappa^{2}}\left(h_{-i \mathbf{n} \mathbf{m}}\right)^{*}\left(\partial^{2}-\mu_{\mathbf{n} \mathbf{m}}^{2}\right) h_{-i \mathbf{n} \mathbf{m}}\right. \\
& +\frac{1}{8}\left(V_{+i \mathbf{n} \mathbf{m}}\right)^{*}\left(\partial^{2}-\mu_{\mathbf{n} \mathbf{m}}^{2}\right) V_{+i \mathbf{n} \mathbf{m}}+\frac{1}{8}\left(V_{-i \mathbf{n} \mathbf{m}}\right)^{*}\left(\partial^{2}-\mu_{\mathbf{n} \mathbf{m}}^{2}\right) V_{-i \mathbf{n} \mathbf{m}} \\
& -\frac{2 \mu_{\mathbf{n} \mathbf{m}} i}{r_{0} \kappa}\left[\left(h_{+i \mathbf{n} \mathbf{m}}\right)^{*} V_{i} \mathbf{n} \mathbf{m}-\left(h_{-i} \mathbf{n} \mathbf{m}\right)^{*} V_{i} \mathbf{n} \mathbf{m}\right. \\
& \left.+\frac{\kappa}{2} \gamma\left(\left(V_{+i \mathbf{n} \mathbf{m}}\right)^{*} V_{i \mathbf{n} \mathbf{m}}-\left(V_{-i \mathbf{n} \mathbf{m}}\right)^{*} V_{i \mathbf{n} \mathbf{m}}\right)\right] \\
& \left.+\frac{1}{2}\left(V_{i \mathbf{n} \mathbf{m}}\right)^{*}\left(\partial^{2}-\mu_{\mathbf{n} \mathbf{m}}^{2}-\frac{8 \gamma^{2}}{r_{0}^{2}}\right) V_{i \mathbf{n} \mathbf{m}}\right\},
\end{aligned}
$$

where the sum over $\mathbf{n}$ and $\mathbf{m}$ is performed over $\mathbf{n}=0,1,2,3, \ldots$ and $\mathbf{m}=0, \pm 1, \pm 2, \pm 3, \ldots$, but with the condition $h_{ \pm i 0,0}=0$ and $V_{ \pm i 0,0}=0$. Also, as a consequence of the reality conditions $h_{+i}(X)=h_{-i}^{*}(X), V_{+i}(X)=V_{-i}^{*}(X)$ and $V_{i}(X)=V_{i}^{*}(X)$, we have the relations $h_{+i \mathbf{n} \mathbf{m}}(x)=h_{-i \mathbf{n}-\mathbf{m}}^{*}(x), V_{+i \mathbf{n} \mathbf{m}}(x)=V_{-i \mathbf{n}-\mathbf{m}}^{*}(x)$ and $V_{i \mathbf{n} \mathbf{m}}(x)=V_{i \mathbf{n}-\mathbf{m}}^{*}(x)$.

In this way, the squared mass operator has finally been transformed into an algebraic matrix with constant entries and we can find its eigenvalues exactly.

6D EYM $\Lambda$ model. To address the spin-1 fluctuations in the 6D EYM $\Lambda$ model, we set $\gamma=0$ and remove the Kalb-Ramond perturbations $\left(V_{ \pm i}=0\right)$ in the above $4 \mathrm{D}$ bilinear action, eq. (5.33). By diagonalizing the corresponding mass-matrix, we find that the explicit helicity- $( \pm 1)$ towers are as follows

$$
M_{\mathbf{n} \mathbf{m}}^{2}=\mu_{\mathbf{n} \mathbf{m}}^{2} \geq 0,
$$

with $\mathbf{n}=0,1,2,3, \ldots$ and $\mathbf{m}=0, \pm 1, \pm 2, \pm 3, \ldots$, but $0 \nless|\mathbf{m}| \omega \nless 1$ and

$$
M_{\mathbf{n} \mathbf{m}}^{2}=\mu_{\mathbf{n} \mathbf{m}}^{2} \pm \frac{2 \sqrt{2}}{r_{0}} \mu_{\mathbf{n} \mathbf{m}} \geq 0
$$

with $\mathbf{n}=0,1,2,3, \ldots$ and $\mathbf{m}=0, \pm 1, \pm 2, \pm 3, \ldots$, but $0 \nless|\mathbf{m}| \omega \nless 1$ and $\{\mathbf{n}, \mathbf{m}\} \neq\{0,0\}$. Neither tachyons nor ghosts are found. The $\{\mathbf{n}, \mathbf{m}\}=\{0,0\}$ mode in (5.34) is the massless

\footnotetext{
${ }^{23}$ It is easy to show that all we have stated about the harmonic expansion for $h_{m i}$ holds for $V_{m i}$ as well.
} 


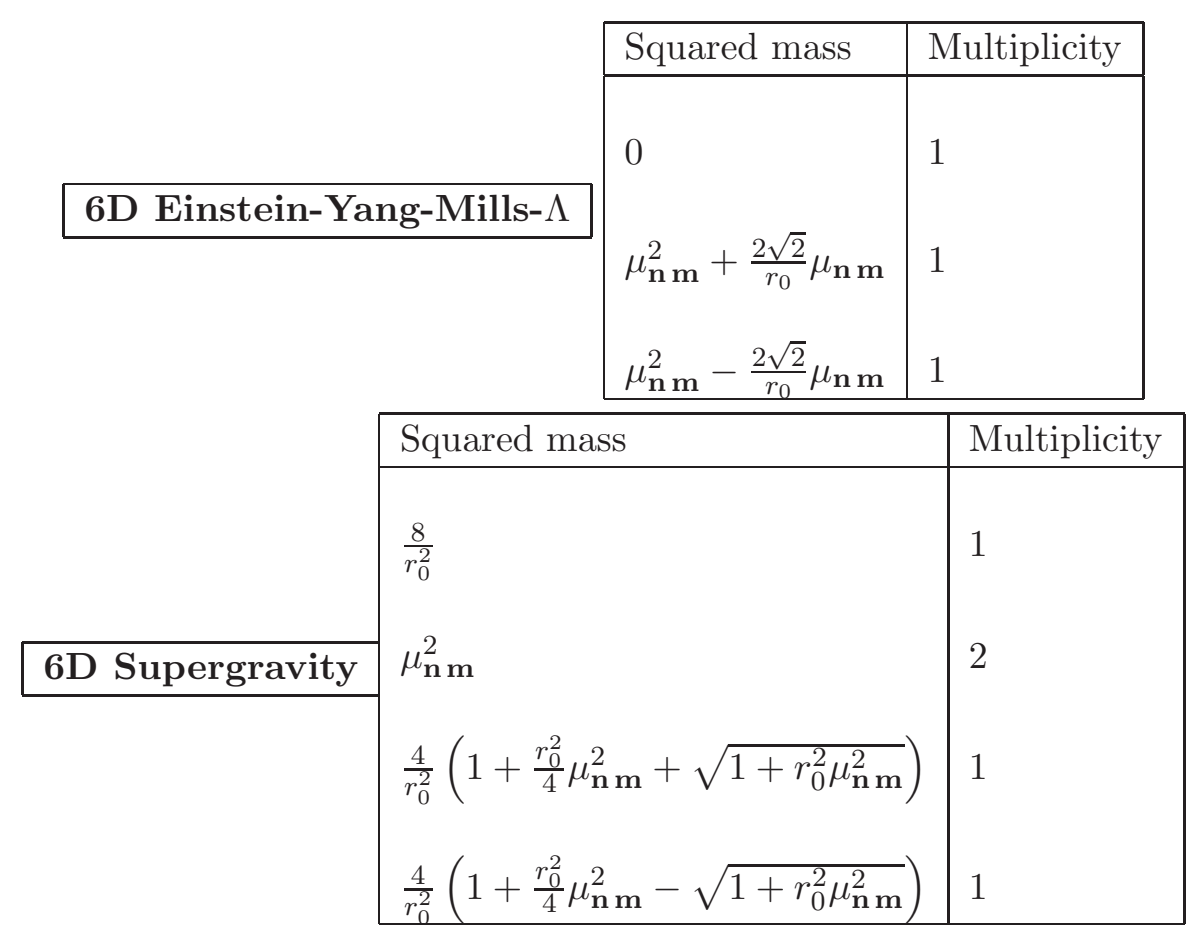

Table 1. Squared mass KK towers of physical spin-1 perturbations around the rugbyball solution to the $6 \mathrm{D}$ EYM $\Lambda$ model and $6 \mathrm{D}$ supergravity, for modes satisfying (5.31). $\mu_{\mathbf{n} \mathbf{m}}^{2}$ is defined in (5.28), but here the KK numbers $\mathbf{n}, \mathbf{m}$ run over $\mathbf{n}=0,1,2,3, \ldots, \mathbf{m}=0, \pm 1, \pm 2, \pm 3, \ldots$, with the constraint $\{\mathbf{n}, \mathbf{m}\} \neq\{0,0\}$.

gauge field associated with the $6 \mathrm{D}$ monopole $\mathrm{U}(1)$, which we have previously discussed in (5.27). The remaining modes in (5.34) are instead the helicity- $( \pm 1)$ components of massive gravitons; we observe that the massive part of the KK tower (5.13) is exactly reproduced by (5.34), according to $4 \mathrm{D}$ Poincaré invariance. The KK towers in (5.35) correspond instead to physical spin-1 fields. The complete set of masses for physical spin-1 fields is given in table 1.

By analyzing those towers, one easily finds that there are physical massless $4 \mathrm{D}$ spin- 1 fields (in addition to (5.27)) if and only if $\mu_{\mathbf{n} \mathbf{m}}^{2}=8 / r_{0}^{2}$, which can be restated as

$$
\{\mathbf{n}, \mathbf{m} \omega\}=\{1,0\} \quad \text { or } \quad\{\mathbf{n}, \mathbf{m} \omega\}=\{0, \pm 1\} .
$$

Therefore there is at least one massless spin-1 field for any value of the tension (this corresponds to $\{\mathbf{n}, \mathbf{m}\}=\{1,0\})$. In the sphere case $(\omega=1)$ we have three ways to satisfy this condition, that is

$$
\{\mathbf{n}, \mathbf{m}\}=\{1,0\},\{0, \pm 1\},
$$

which correspond to the three gauge fields of $\mathrm{SU}(2)_{K K}$, whereas for positive tension rugbyballs and generic saddle-spheres there is only one choice:

$$
\{\mathbf{n}, \mathbf{m}\}=\{1,0\}
$$


corresponding to the KK gauge group $\mathrm{U}(1)_{K K}$. However, for the special saddle-spheres for which (5.17) holds, the number of massless vector fields is enhanced from one plus one to one plus three!

We shall discuss in detail the physical significance of these modes in the following subsection.

$6 \mathrm{D}$ supergravity. We conclude this subsection by providing the helicity- $( \pm 1)$ masses for the 6D supergravity (set $\gamma=1$ and keep the Kalb-Ramond fluctuations in eq. (5.33)). Diagonalizing the corresponding mass-matrix, we find:

$$
M^{2}=\frac{8}{r_{0}^{2}}>0
$$

which is the vector field associated with the monopole $\mathrm{U}(1)$,

$$
M_{\mathbf{n} \mathbf{m}}^{2}=\mu_{\mathbf{n} \mathbf{m}}^{2} \geq 0, \quad \text { with multiplicity } 3
$$

and

$$
M_{\mathbf{n} \mathbf{m}}^{2}=\frac{4}{r_{0}^{2}}\left(1+\frac{r_{0}^{2}}{4} \mu_{\mathbf{n} \mathbf{m}}^{2} \pm \sqrt{1+r_{0}^{2} \mu_{\mathbf{n} \mathbf{m}}^{2}}\right) \geq 0
$$

where $\mathbf{n}=0,1,2,3, \ldots, \mathbf{m}=0, \pm 1, \pm 2, \pm 3, \ldots,\{\mathbf{n}, \mathbf{m}\} \neq\{0,0\}$ and $0 \nless|\mathbf{m}| \omega \nless 1$. The masses in (5.37), two towers out of three in (5.38) and the towers in (5.39) correspond to physical spin-1 fields, whereas one of the towers in (5.38) are the helicity- $( \pm 1)$ components of massive gravitons. The complete set of masses for the physical spin-1 fields is summarized in table 1. We note that neither tachyons nor ghosts are found.

Regarding the massless vector fields the situation is similar to the 6D EYM $\Lambda$ model. It easy to see that the condition for masslessness is again (5.36) and, therefore, again we have a single KK massless gauge boson for positive tensions and generic negative tensions; instead, for negative tensions of the form (5.17), the number of massless vector fields is enhanced from one to three!

As an effective check of the results presented in this subsection, we have also derived the aforementioned spectrum in the sphere case $(\omega=1)$ by expanding the bulk fields over the Wigner functions as in [31] and obtained exactly the sphere limit of our towers.

\subsection{Massless vectors and 4D gauge symmetries}

In the previous subsection we observed three massless 4D vector fields amongst the KK spectra for the $6 \mathrm{D}$ models $^{24}$ on both the sphere and the special saddle-spheres (5.17). We shall now address the physical significance of these modes. One of them, the one with axial quantum number $\mathbf{m}=0$, should provide the gauge boson for the $\mathrm{U}(1)_{K K}$ gauge symmetry, descending from the axial isometry of the internal space. The other two massless vectors, having $\mathbf{m} \neq 0$, are charged under this axial symmetry and so we may expect the three vectors to fit into a non-Abelian structure, like $\mathrm{SU}(2)$. For the sphere, this is indeed the case, and the three massless vectors compose the gauge fields of an $\mathrm{SU}(2)$ gauge symmetry

\footnotetext{
${ }^{24}$ In the EYM $\Lambda$ model there is also a massless vector field descending from the higher dimensional $\mathrm{U}(1)$ gauge field, which forms a $4 \mathrm{D} \mathrm{U}(1)$ gauge field.
} 
in the $4 \mathrm{D}$ theory. What happens for the special saddle-spheres, where there is no $\mathrm{SU}(2)$ isometry in the background? Let's take $\alpha=2,3, \ldots$, so we consider the special saddlespheres (we also allow for the smooth sphere with $\alpha=1$ ).

\subsubsection{Why there are three massless vector modes}

Let us begin by understanding why three massless vector modes appear in the spectrum, despite the fact that any branes clearly break the $\mathrm{SU}(2)$ isometries of the sphere.

Above, we found that the massless vector fields arise as a linear combination of $h_{\mu \mathbf{m n}}^{m}(x)$ and $V_{\mu \mathbf{m n}}(x)$ (and $V_{\mu}^{m} \mathbf{m n}(x)$ for $6 \mathrm{D}$ supergravity), once we have integrated out the extra dimensions. In detail, if one takes the squared mass matrix defined implicitly by the $4 \mathrm{D}$ bilinear action in (5.33) in e.g. the EYM $\Lambda$ case, one finds that the mass eigenstates are $(\{\mathbf{n}, \mathbf{m}\} \neq(0,0))$ :

$$
\begin{aligned}
A_{i} \mathbf{n m} & =\frac{i}{2} h_{+i} \mathbf{n m}-\frac{i}{2} h_{-i} \mathbf{n m}+\frac{1}{\sqrt{2}} V_{i} \mathbf{n m} \\
U_{i} \mathbf{n m} & =-\frac{i}{2} h_{+i \mathbf{n m}}+\frac{i}{2} h_{-i \mathbf{n m}}+\frac{1}{\sqrt{2}} V_{i} \mathbf{n m} \\
W_{i \mathbf{n m}} & =\frac{1}{\sqrt{2}} h_{+i} \mathbf{n m}+\frac{1}{\sqrt{2}} h_{-i \mathbf{n m}}
\end{aligned}
$$

corresponding respectively to $M_{\mathbf{n} \mathbf{m}}^{2}=\mu_{\mathbf{n} \mathbf{m}}^{2}-\left(2 \sqrt{2} / r_{0}\right) \mu_{\mathbf{n} \mathbf{m}}, M_{\mathbf{n} \mathbf{m}}^{2}=\mu_{\mathbf{n} \mathbf{m}}^{2}+\left(2 \sqrt{2} / r_{0}\right) \mu_{\mathbf{n} \mathbf{m}}$ and $M_{\mathbf{n} \mathbf{m}}^{2}=\mu_{\mathbf{n} \mathbf{m}}^{2}$ in (5.35) and (5.34). Recall that the massless modes emerge from the $A_{i \mathbf{n m}}$ tower, when $\{\mathbf{n}, \mathbf{m} \omega\}=\{1,0\},\{0, \pm 1\}$.

We can write, then, the harmonic expansion of $h_{\mu}^{m}(X)$ as:

$$
h_{\mu}^{m}(X)=\sum_{I=-, 0,+} A_{\mu}^{I}(x) K^{I m}(\theta, \varphi)+\text { massive modes } .
$$

Using the expansion (5.29), (5.30), the explicit form for the wave functions (5.32) and the rearrangement in terms of the mass eigenstates (5.40), it is straightforward to show that $K^{I m}(\theta, \varphi)$ indeed correspond to the Killing vectors (5.18) on the special saddle-sphere, where $I=0$ corresponds to $\{\mathbf{n}, \mathbf{m} \omega\}=\{1,0\}$ and $I= \pm$ to $\{\mathbf{n}, \mathbf{m} \omega\}=\{0, \pm 1\}$. This is just as in the traditional KK reduction scheme.

In this way, we confirm that the presence of infinitesimal isometries on the internal space, which are generated by Killing vector fields, is sufficient for the appearance of massless vector modes - even if they cannot be integrated to genuine isometries.

\subsubsection{The absence of enhanced gauge symmetries in the full $4 \mathrm{D}$ theory}

We now ask whether or not these massless vector modes behave as gauge fields of an $\mathrm{SU}(2)$ gauge symmetry. The linearized 4D theory cannot probe any non-Abelian structure, and so to understand the gauge invariance of the full $4 \mathrm{D}$ theory, we must go beyond linear order. To this end, we consider a simple extension of the EYM $\Lambda$ model, where we add a single complex, massless, neutral scalar field which has an action:

$$
S_{\Phi}=-\int d^{6} X \sqrt{-G} \partial_{M} \Phi^{*} \partial^{M} \Phi
$$


and which assumes a trivial VEV in the saddle-sphere background. It is easy to see that the linearized equation of motion for $Z:=\delta \Phi$ gives rise to the rugbyball scalar harmonics (see above eq. (5.28)):

$$
Z(X)=\sum_{\mathbf{m}, \mathbf{n}} z_{\mathbf{m n}}(x) f_{\mathbf{m n}}(\theta) e^{i \mathbf{m} \varphi}
$$

with the corresponding masses (5.28):

$$
M^{2}=\frac{4}{r_{0}^{2}} l(l+1) \quad \text { where } \quad l=\mathbf{n}+|\mathbf{m}| \omega .
$$

The multiplicity of a given mass is given by $2 l+1$ when $l$ is integer or half-odd integer; otherwise it is given by $2\left([l]+\frac{1}{2}\right)+1$, where $[l]$ denotes the integer part of $l$. We also note that for $l$ integer (which corresponds also to $\mathbf{m} \omega$ integer), the wavefunction $f_{\mathbf{m n}}(\theta)$ is an Associated Legendre function, just as for the spherical harmonics. The modes with $l$ non-integer are instead additional harmonics, which generically have no corresponding states amongst the spherical harmonics nor indeed any of the Wigner functions.

Now let us ask how the $4 \mathrm{D}$ fields $z_{\mathbf{m n}}(x)$ couple to the massless vector fields, and in particular if they do in a gauge-invariant way. At trilinear level, this coupling descends only from the term:

$$
S\left(Z^{*}, h_{\mu m}, Z\right)=-\int d^{6} X \sqrt{-G} \partial^{\mu} Z^{*} h_{\mu}^{m} \partial_{m} Z
$$

and its complex conjugate. The above trilinear coupling can now be reexpressed in terms of the $4 \mathrm{D}$ fields, and isolating the contributions involving the massless vectors, eq. (5.41), we find:

$$
S\left(z^{*}, A_{\mu}^{I}, z\right)=-\int d^{4} x \sqrt{-g_{4}} \partial^{\mu} z^{*} A_{\mu}^{I} z^{\prime} \int d \theta d \varphi \frac{r_{0}^{2}}{4} \alpha \sin \theta f e^{-i \mathbf{m} \varphi} K^{I m} \partial_{m}\left(f^{\prime} e^{i \mathbf{m}^{\prime} \varphi}\right),
$$

where we have suppressed the KK indices $\{\mathbf{n}, \mathbf{m}\}$ and $\left\{\mathbf{n}^{\prime}, \mathbf{m}^{\prime}\right\}$ on $z, f$ and $z^{\prime}, f^{\prime}$ respectively. Performing the integral over the internal dimensions:

$$
g_{I}=\int d \theta d \varphi \frac{r_{0}^{2}}{4} \alpha \sin \theta f e^{-i \mathbf{m} \varphi} K^{I m} \partial_{m}\left(f^{\prime} e^{i \mathbf{m}^{\prime} \varphi}\right),
$$

we see that the wavefunction overlap (5.47) gives the $4 \mathrm{D}$ coupling between $z(x)$ and $z^{\prime}(x)$ via a massless vector field, $A_{\mu}^{I}(x)$ :

$$
-g_{I} \int d^{4} x \sqrt{-g_{4}} \partial^{\mu} z^{*} A_{\mu}^{I} z^{\prime}
$$

Observe that if the full $4 \mathrm{D}$ theory were to respect an $\mathrm{SU}(2)$ gauge symmetry whose gauge fields are the three massless vector modes, then $z$ and $z^{\prime}$ would belong to $\mathrm{SU}(2)$ multiplets of the same dimension and (5.48) would correspond to the trilinear terms in the gauge invariant combination $-D_{\mu} z^{a *} D^{\mu} z^{\prime a}$, where $D_{\mu} z^{a}=\partial_{\mu} z^{a}+A_{\mu}^{I} T_{b}^{I a} z^{b}$, the indices $a, b$ run over $a, b=1, \ldots, r$ and $r$ is the size of the multiplet. For the classic sphere, $\alpha=1$, this is of course the case, and the wave function overlaps in (5.47) are zero unless $z$ and $z^{\prime}$ 
belong to the same $\mathrm{SU}(2)$ multiplet, thanks to the properties of the spherical harmonics. We shall now see that such a structure does not hold for the special saddle-spheres.

To this purpose, let us consider the rugbyball harmonics with $0<\mathbf{m} \omega<1$. The pattern that emerges for the overlaps (5.47) once both the integrals over $d \theta$ and $d \phi$ are performed, ${ }^{25}$ is that a mode, $f$, with $0<\mathbf{m} \omega<1$ and $\mathbf{n}$ even (respectively odd) has a non-zero overlap with the modes, $f^{\prime}$, for which $\mathbf{m}^{\prime} \omega=\mathbf{m} \omega \mp 1$ and all $\mathbf{n}^{\prime}$ odd (respectively even). It can then easily be seen that this prevents the realization of an $\mathrm{SU}(2)$ gauge symmetry. Take for instance the set of modes $\{z\}$ with some mass-squared $l(l+1)$ in which $0<|\mathbf{m}| \omega<1$ and $\mathbf{n}=0$. This mass comes only with degeneracy 2, corresponding to KK numbers $\{0, \pm \mathbf{m}\}$. Therefore, if there exists an $\mathrm{SU}(2)$ gauge symmetry, then the modes in $\{z\}$ fall either into an $\mathrm{SU}(2)$ doublet or two singlets. The overlap (5.47) between the modes $\{0, \mathbf{m}\}$ and $\{0,-\mathbf{m}\}$ is zero, and the subsequent vanishing of the coupling in (5.48) tells us that $\{z\}$ cannot form a doublet. On the other hand, the modes $\{0, \pm \mathbf{m}\}$ do have a non-zero overlap with $\{\mathbf{n}$ odd, $\pm \mathbf{m}-1 / \omega\}$ and $\{\mathbf{n}$ odd, $\pm \mathbf{m}+1 / \omega\}$, and so the two modes in $\{z\}$ each have a trilinear coupling (5.48) with towers of $z^{\prime}$ and the massless vectors fields. Thus, they cannot be singlets. In this way we can conclude that there does not exist an $\mathrm{SU}(2)$ gauge symmetry corresponding to the massless vector fields.

We would like to draw one more insight into the absence of $\mathrm{SU}(2)$ gauge symmetry for the full $4 \mathrm{D}$ theory. The Killing vectors (5.18) can be considered as generators of an $\mathrm{SU}(2)$ algebra, and the mass-squared operator for the saddle-sphere scalars, $-D^{2}$, can be understood as the Casimir Operator for the algebra: $-\frac{r_{0}^{2}}{4} D^{2}=\frac{1}{2}\left(K^{+} K^{-}+K^{-} K^{+}\right)+$ $\left(K^{0}\right)^{2}$. The saddle-sphere scalar harmonics form a basis for the Hilbert space of functions on which the Hermitian operator, $-D^{2}$ (plus boundary conditions), acts. However, the $\mathrm{SU}(2)$ ladder operators, $K^{ \pm}$, do not act within this Hilbert space: the action of $K^{ \pm}$on the harmonics $f_{\mathbf{m n}}(\theta) e^{i \mathbf{m} \varphi}$ with $0<|\mathbf{m}| \omega<1$ gives back functions which do not obey the $\mathrm{NC}$ and $\mathrm{HC}$ boundary conditions. Again, we see that the saddle-sphere harmonics do not furnish well-defined representations of the $\mathrm{SU}(2)_{K K}$ generated by the Killing vectors, and it is precisely the modes with $0<|\mathbf{m}| \omega<1$ that are the problematics ones. ${ }^{26}$

As we have implied above, the absence of an SU(2) KK gauge symmetry in the $4 \mathrm{D}$ theory can be understood in the $6 \mathrm{D}$ picture as being due to the absence of a genuine $\mathrm{SU}(2)$ isometry in the internal dimensions.

\subsubsection{The emergence of enhanced gauge symmetries at low energies}

Finally, notice that although the modes with $0<|\mathbf{m}| \omega<1$ do not belong to well-defined $\mathrm{SU}(2)$ representations, the massless wave functions that we have found are equivalent to those present in the sphere case (up to an integer constant multiplying $\varphi$ ) and do furnish

\footnotetext{
${ }^{25}$ Whilst we have not checked this result for all $\omega, \mathbf{m}$ and $\mathbf{n}$ the pattern is quite convincing.

${ }^{26}$ Notice that this range of $\mathbf{m}$ is empty for the special saddle-sphere with $\omega=\frac{1}{2}$ if we impose the $Z_{2}$ orbifold projection discussed in appendix C. In this case, then, all the KK modes are in well-defined representations of $\mathrm{SU}(2)$ (corresponding to the Wigner functions), and we can expect an $\mathrm{SU}(2)$ gauge invariance in the full $4 \mathrm{D}$ theory, at least if we remove the branes and discuss a smooth non-compact manifold. This is not surprising, since - outside the branes - the $Z_{2}$ orbifolding effectively cancels out the $\delta=-2 \pi$ deficit angle, and we return to the standard sphere case.
} 
well-defined SU(2) representations. ${ }^{27}$ This holds also for the massless spin-2 and spin-1 fields above, as well as the massless spin-0 fields discussed below. ${ }^{28}$ Therefore, the classical low energy $4 \mathrm{D}$ effective theory that results from truncating the massive modes does enjoy an $\mathrm{SU}(2) \mathrm{KK}$ gauge invariance to all orders in perturbation theory - despite the absence of a genuine $\mathrm{SU}(2)$ isometry in the extra dimensions. Indeed, this low energy $4 \mathrm{D}$ theory does not distinguish between a compactification on a smooth sphere or a special saddle-sphere!

Moreover, we can argue that the above truncation to the massless sector is a consistent one, ${ }^{29}$ at least for the bosonic theory that we have studied whose field content is identical to that of $6 \mathrm{D}$ supergravity. Then, if we remove the branes and replace the singular space with a smooth non-compact manifold, the local geometry is the same for the sphere everywhere and the KK ansatz for the special saddle-sphere is essentially identical to that of the smooth sphere. Meanwhile, the sphere reduction of $6 \mathrm{D}$ supergravity was shown to be a consistent one in ref. [35], thanks to a remarkable conspiracy between properties of the 2-sphere and the structure of supergravity.

\subsection{Massless scalars}

Finally, we turn to the spectra of $4 \mathrm{D}$ spin-0 fields, which are governed by the action given in appendix B. In appendix $\mathrm{C}$ we give the complete spectra for unwarped braneworld compactifications in 6D supergravity. Here, our focus shall be on the massless scalars featured in the low energy 4D effective theory. Again we shall first review the RS model and then examine the $6 \mathrm{D}$ braneworld models.

\subsubsection{Randall-Sundrum}

In the RS model of ref. [1], the massless scalar sector involves one normalizable mode (the radion), which becomes non normalizable in the decompactification limit $r_{c} \rightarrow \infty$ [29]. Let us find this mode in our formalism. We can of course restrict our attention to the spin-0 action $S^{(0)}(h, h)$ as in [1] only gravity is introduced and the branons are consistently projected out by the $S^{1} / Z_{2}$ orbifold conditions. Therefore, we only have to deal with the perturbation $h_{\rho \rho}$, because (4.2) implies $h_{i}{ }^{i}=-h_{\rho \rho}$. It is easy to derive the EOM for $h_{\rho \rho}$ :

$$
-\frac{1}{\sqrt{-G}} \partial_{M}\left(\sqrt{-G} \partial^{M} h_{\rho \rho}\right)+\left[-A^{\prime 2}+\frac{1}{3} T \kappa^{2} \delta(\rho-Y)\right] h_{\rho \rho}=0,
$$

where $T \kappa^{2} \delta(\rho-Y) \equiv T_{1} \kappa^{2} \delta(\rho)+T_{2} \kappa^{2} \delta\left(\rho-\pi r_{c}\right)$. We now perform a KK decomposition $h_{\rho \rho}(x, \rho)=\sum_{\mathbf{n}} h_{\rho \rho}(x) f_{\mathbf{n}}(\rho)$ and focus on the massless case $\left(\eta^{\mu \nu} \partial_{\mu} \partial_{\nu}=0\right)$; we obtain the simple equation

$$
\psi^{\prime \prime}=0,
$$

\footnotetext{
${ }^{27}$ This is a consequence of the fact that in our mass-squared's, $M^{2}$, as well as in our $f_{\mathbf{n m}}(\theta)$ wavefunctions, $\mathbf{m}$ and $\omega$ enter only through the combination $\mathbf{m} \omega$. This is obvious for the masses, and for the wave functions it can be seen from eq. (5.11), after setting $\bar{\omega}=\omega$ to recover the spectra for the rugbyball. Furthermore, the massless modes all have integer $\mathbf{m} \omega$.

${ }^{28}$ We should caution that, although there are no symmetries that suggest them to be massless, our harmonic analysis has not included the modes with $0<|\mathbf{m}| \omega<1$ in the spin-1 sector, nor the modes with $0<|\mathbf{m}| \omega<1$ and $1<|\mathbf{m}| \omega<2$ in the spin-0 sector.

${ }^{29}$ Mathematical consistency may of course not be necessary, if the truncation is consistent up to some energy scale.
} 
where we have defined $\psi \equiv e^{A} f$ and used property (5.16). The only solution to (5.50) satisfying the $S^{1} / Z_{2}$ orbifold conditions is $\psi$ constant, which corresponds to

$$
f \propto e^{-A}
$$

Mode (5.51) is the wave function of the radion. By inserting this mode in the kinetic term of $h_{\rho \rho}$ in (B.1) one easily finds that it is normalizable for any finite $r_{c}$, but becomes non normalizable in the limit $r_{c} \rightarrow \infty$.

\subsubsection{D brane worlds}

After this non-trivial check of our formalism we now turn to the conical-GGP solutions of $6 \mathrm{D}$ supergravity. The stability of the GGP solutions has been investigated in [7] and [9], where no tachyons emerged unless non-Abelian gauge groups are considered. Indeed, in the presence of non-Abelian gauge groups, an instability may arise in the sector described by the action $S^{(0)}(V, V)$, with $V_{\underline{m}}$ orthogonal to the background monopole ${ }^{30}$ [9]. We observe that, even in the absence of non-Abelian gauge groups, the stability of the GGP solutions is marginal, in the sense that there are necessarily massless scalars in the physical spectrum. These massless particles are manifestations of two symmetries in the model. One is the following invariance of the EOMs: $G_{M N} \rightarrow w G_{M N}$ and $e^{\phi / 2} \rightarrow w e^{\phi / 2}$, where $w$ is a real number. Note that this is only a classical symmetry because the action rescales as $S_{B} \rightarrow w^{2} S_{B}$, so we do not expect the corresponding scalar to remain massless once quantum corrections are included. The other is the Kalb-Ramond symmetry, which acts as $B_{2} \rightarrow B_{2}+d \Lambda$, where $\Lambda$ is a general 1-form field. The actual presence of the zero mode corresponding to the former symmetry has been shown in refs. $[7,11]$.

Here, by using our bilinear action, we can easily figure out where the other massless scalar is. This emerges as the lightest $4 \mathrm{D}$ mode of the field $V_{i j}$, whose bilinear action is simply (see eq. (B.7))

$$
-\frac{1}{16} \int d^{6} X \sqrt{-G} e^{\phi-2 A} \partial_{M} V_{i j} \partial^{M} V_{i j} .
$$

This action is equivalent to the spin-2 action (4.4) in the case of the conical-GGP solutions, which satisfy $A=\phi / 2$. The wave functions and mass spectrum coming from $V_{i j}$ are therefore identical to the one presented in Subsection 5.1. For $\mathbf{n}=\mathbf{m}=0$ we obtain the massless scalar field associated to the Kalb-Ramond symmetry. In the spherical limit this corresponds to the $l=0$ mode in (5.14) [27].

\section{$6 \quad$ Summary of results}

Before concluding, let us provide an overview of our results.

- We have derived the linearized dynamics, eqs. (4.4)-(4.9) and appendix B, for the physical perturbations about general backgrounds in a general class of field theories. In particular, we take Einstein-Yang Mills (EYM) theory in $D$ spacetime dimensions,

\footnotetext{
${ }^{30}$ This instability is also present in the sphere-monopole solution [34], which is a particular case of the GGP solutions.
} 


\begin{tabular}{|l|c|c|c|}
\hline & spin- 2 & spin-1 & spin-0 \\
\hline Rugby-ball $\delta \geq 0$ & all modes & all modes & all modes \\
Saddle-sphere $\delta=-2 \pi$ & all modes & all modes & all modes \\
Generic Saddle-sphere & all modes & $\mathbf{m}=0 ;|\mathbf{m}| \geq 1 / \omega$ & $|\mathbf{m}|=0,1 / \omega ;|\mathbf{m}| \geq 2 / \omega$ \\
Warped Models & all modes & - & - \\
\hline
\end{tabular}

Table 2. The sectors covered in the present paper for Braneworld Compactifications in 6D Supergravity. In order to address the spin-0 sector, we projected out the branons with an orbifolding. We also here impose the orbifolding for all sectors in the presence of negative tension branes.

with a bulk dilaton or cosmological constant $(\Lambda)$, and a number of dynamical 3branes. Moreover, for $D=6$ we include a dilaton and 2-form potential. Therefore, 6D chiral supergravity, D-dimensional EYM $\Lambda$ theory and the 5D Randall-Sundrum models all fall within our analysis. The backgrounds considered respect 4D Poincaré invariance, but may be warped in a radial transverse coordinate.

- Taking the Randall-Sundrum models as an illustrative example within our formalism, we retrieve the well-known dynamics for spin-2 fluctuations and identify the massless scalar (the radion), which is normalizable in the two brane model and becomes nonnormalizable in the one brane model.

- For the 6D EYM $\Lambda$ model, we consider the unwarped "rugbyball-monopole" compactifications, sourced by two 3-branes of equal tension. When the tensions are zero, we recover the sphere-monopole compactification, and when the tensions are negative we refer to the 2D geometry as a "saddle-sphere". By deriving a set of "rugbyball harmonics", we are able to obtain analytic KK spectra; i.e. we discuss how to find physical 4D spin-2, spin-1 and - consistently truncating branons - spin-0 fields and their masses. We present the full spin- 2 spectrum and the spin-1 spectra for axial momentum number $0 \nless|\mathbf{m}| \omega \nless 1$.

- For the $6 \mathrm{D}$ supergravity, the backgrounds of interest are the warped, axially symmetry braneworld ("conical-GGP") solutions, which have unwarped limits to the rugyballs and saddle-spheres, and to the sphere. Our focus is on the bosonic "Salam-Sezgin" sector (from the gravity-tensor supermultiplet and the U(1) gauge multiplet in which the background monopole lies), since the remaining bosonic sectors have been treated elsewhere. We obtain the complete spin- 2 spectrum. For the spin- 1 and spin- 0 sectors, we restrict to the unwarped backgrounds, and employ the rugbyball harmonics to find the spectra. The sectors covered by our analysis ${ }^{31}$ are summarized in detail in table 2 .

Our main physical results for the $6 \mathrm{D}$ braneworlds are as follows.

- The spin-2 spectrum includes the massless 4D graviton separated from the rest of the KK tower by a mass gap, and the mass gap is indeed observed in all sectors. For

\footnotetext{
${ }^{31}$ We also find the spectrum in the sphere case as a check.
} 
rugbyballs with positive deficit angles and for generic saddle-spheres, the spin-1 sector contains a massless KK gauge boson due to the $\mathrm{U}(1)$ isometry in the background (in addition to any massless 4D gauge bosons descending from unbroken $6 \mathrm{D}$ gauge symmetries). For the special saddle-spheres with deficit angles $\delta=-2 \pi,-4 \pi, \ldots$, there is a qualitative difference. Here, there are three Killing vectors, which are welldefined everywhere outside the branes and obey an SU(2) Lie algebra. Although only one of them integrates to a genuine isometry, the number of massless KK vectors fields is consequently enhanced to three. Meanwhile, in the spin-0 sector for supergravity, we identify the two massless scalar fields expected in all cases from the classical scaling symmetry and the Kalb-Ramond symmetry.

- The spin-2 and spin-1 spectra are all well-behaved despite the presence of codimension-two dynamical brane sources, which induce singularities in the bulk geometry. To make progress in the spin-0 sector, we had to discard the branon modes (e.g. by placing the branes at orbifold fixed points).

- The spectra analysed - which incorporates all modes for rugbyballs sourced by positive tension branes - do not harbour any instabilities; neither tachyons nor ghosts.

- To understand the significance of the three massless 4D vector fields that appear for the special saddle-spheres, we go beyond bilinear order. We find that in the full 4D theory, they do not represent gauge fields of an $\mathrm{SU}(2)$ gauge symmetry. This is due to the presence of KK modes that are not in well-defined $\mathrm{SU}(2)$ representations. The classical masslessness of the vector fields is thus not protected by any symmetry, which is in accordance with the absence of a genuine $\mathrm{SU}(2)$ isometry in the background.

- In the massless sector, however, all modes fall into well-defined $\mathrm{SU}(2)$ representations. Therefore, the low energy $4 \mathrm{D}$ effective theory obtained by truncating to the massless sector does seem to enjoy a classical $\mathrm{SU}(2) \mathrm{KK}$ gauge symmetry, despite the absence of a background $\mathrm{SU}(2)$ isometry! Indeed, this low energy effective theory does not distinguish between compactifications on the sphere and the special saddle-spheres.

\section{Conclusions}

In this paper, we have provided the dynamics of the physical fluctuations in a wide class of models, which incorporate the bosonic fields generically present in bulk supergravity theories - gravity, non-Abelian gauge fields, the dilaton and two-form potential - as well as dynamical 3-branes. Our final equations ((4.4)-(4.9) and those in appendix B), which can be considered as a generalization of the analysis in [12], provide the starting point to construct a $4 \mathrm{D}$ effective field theory emerging from various higher dimensional models, with compactified extra dimensions and/or branes.

We next proceeded with that objective to study the behaviour of braneworlds solutions in six dimensions, taking as representative the rugbyball compactifications of EinsteinYang Mills theory with a cosmological constant (EYMA) and certain axi-symmetric warped compactifications to 6D minimal gauged supergravity; the so-called conical-GGP solutions. 
We have obtained the complete KK spectrum for the 4D spin-2 sector in the conical-GGP solutions, which is a step towards understanding the behaviour of gravity in codimension two braneworld models, as for example studied in [36]. The spin-1 and spin-0 sectors present large systems of coupled differential equations to be solved (five-by-five for the spin-1 fluctuations, eight-by-eight for the spin-0 fluctuations after truncating the branons), and we are able to do so in the unwarped cases by developing "rugbyball harmonics", in analogy to the spherical harmonics. Along the way, we also recovered some familiar features of the 5D Randall-Sundrum models. Our main results are summarized in the previous section.

Previous studies have revealed that codimension-two braneworld compactifications can evade the traditional KK lore in several ways. For instance, in [6] it was found that the KK mass-gap can be decoupled from the size of the extra dimensions in the presence of negative tension branes, in principle allowing not only gravity but also the SM to propagate in large extra dimensions. This phenomenon can also be observed here. We can also now suggest the following. The power-law warping present in the $6 \mathrm{D}$ braneworlds studied here does not change qualitatively the physics. Moreover, models with only positive tension codimension-two branes also have qualitatively the same behaviour as traditional KK compactifications. Meanwhile, the introduction of negative tension codimension-two branes can lead to surprising dynamics.

As yet another example of how the physics of braneworlds in $6 \mathrm{D}$ can counter intuition, we have found - for special saddle-sphere compactifications with deficit angles $\delta=-2 \pi,-4 \pi, \ldots$ - three massless vector fields thanks to the presence of three $\mathrm{SU}(2)$ Killing vectors in the internal manifold that are well defined everywhere outside the branes. Thus we see that infinitesimal isometries are sufficient to imply massless vector fields, even if they cannot be integrated to genuine isometries. All the massless modes in the models studied here fall into well-defined representations of the $\mathrm{SU}(2)$, although there are massive KK modes which do not. In this way we see that the massless vectors provide the gauge fields of an enhanced SU(2) KK gauge symmetry in the classical, low energy, 4D effective theory obtained by truncating to the massless sector, despite the absence of an $\mathrm{SU}(2)$ isometry in the background! Apparently, the low energy theory does not distinguish between a compactification on the special saddle-spheres and the smooth sphere.

At the same time, as we approach the energy of the KK mass gap and incorporate the non-zero modes, we see that the $\mathrm{SU}(2) \mathrm{KK}$ gauge symmetry is broken explicitly to $\mathrm{U}(1)$. This is because only the $\mathrm{U}(1)$ is a genuine global continuous isometry of the internal manifold. The masslessness of the extra massless vector fields is thus not protected by any symmetry, and should not survive quantum corrections. Meanwhile, reaching energies far above the KK mass-gap, the full 6D symmetries will be restored as usual. The pattern of symmetry breaking and emergence that we have found within our classical approximation, as different energy scales are probed, is thus a novel one.

In the model whose field content and structure corresponds to the bosonic part of $6 \mathrm{D}$ supergravity, the low-energy theory describes the graviton, the three vectors in the adjoint of $\mathrm{SU}(2)$ and two massless scalars that are $\mathrm{SU}(2)$ singlets. Whether the above properties are shared with fermionic modes is not known and their behaviour, though of interest, lies 
beyond the scope of the present paper. Meanwhile, we argued that we expect the zero-mode truncation to be a consistent one, at least in the aforementioned model once we remove the brane sources and study a non-compact smooth manifold. We thus note that this bosonic model is in principle a complete one, sufficient to demonstrate the unconventional dynamics that we have observed. It would certainly be interesting to check the consistency also in the presence of branes.

This work concludes our study of the bosonic perturbations in the axi-symmetric braneworld solutions to $6 \mathrm{D}$ supergravity. We may now turn to the fermionic sector.

\section{Acknowledgments}

It is a pleasure to thank Cliff Burgess, Claudia de Rham, Stephan Mohrdieck, Nicola Pagani, Riccardo Rattazzi, Michele Redi, Mikhail Shaposhnikov and Andrea Wulzer for valuable discussions. S.L.P. is supported by the Deutsche Forschungsgemeinschaft under the Collaborative Research Center 676 and by the European Union 6th framework program MRTN-CT-503359 "Quest for Unification". A.S. has been supported by the Tomalla Foundation and by CICYT-FEDER-FPA2008-01430. S.L.P. and A.S. thank the High Energy Theory Group at ICTP for hospitality at various stages of this work.

\section{A General $\xi$-dependent bilinear action}

Here we give the explicit expression for the biliner action that depends on the fluctuations of the brane positions $\xi^{M}$, before any gauge fixing, that is the last two terms in (3.5). These terms have been computed by varying the brane action (2.6) with respect to (3.1) and by keeping only terms up to the quadratic order. Their explicit expression is the following

$$
\begin{aligned}
S(\xi, \xi)= & -\frac{T}{2} \int d^{4} x \sqrt{-g}\left[G_{M N} \partial \xi^{M} \cdot \partial \xi^{N}\right. \\
& +\frac{1}{2} \xi^{P} \xi^{R} \partial_{P} \partial_{R} G_{M N} \partial Y^{M} \cdot \partial Y^{N}+2 \xi^{P} \partial_{P} G_{M N} \partial \xi^{M} \cdot \partial Y^{N} \\
& +\frac{1}{2} \xi^{P} \partial_{P} G_{M N} \xi^{R} \partial_{R} G_{S Q}\left(\frac{1}{2} \partial Y^{M} \cdot \partial Y^{N} \partial Y^{S} \cdot \partial Y^{Q}-\partial Y^{M} \cdot \partial Y^{S} \partial Y^{N} \cdot \partial Y^{Q}\right) \\
& +G_{M N} G_{P R}\left(\partial \xi^{M} \cdot \partial Y^{N} \partial \xi^{P} \cdot \partial Y^{R}-2 \partial \xi^{M} \cdot \partial \xi^{P} \partial Y^{N} \cdot \partial Y^{R}\right) \\
& \left.+\xi^{P} \partial_{P} G_{M N} G_{R S}\left(\partial Y^{M} \cdot \partial Y^{N} \partial \xi^{R} \cdot \partial Y^{S}-2 \partial Y^{M} \cdot \partial \xi^{R} \partial Y^{N} \cdot \partial Y^{S}\right)\right],
\end{aligned}
$$

and

$$
\begin{aligned}
S(h, \xi)= & -\frac{T}{2} \int d^{4} x \sqrt{-g}\left[\xi^{P} \partial_{P} h_{M N} \partial Y^{M} \cdot \partial Y^{N}+2 h_{M N} \partial \xi^{M} \cdot \partial Y^{N}\right. \\
& +h_{M N} \xi^{P} \partial_{P} G_{R S}\left(\frac{1}{2} \partial Y^{M} \cdot \partial Y^{N} \partial Y^{R} \cdot \partial Y^{S}-\partial Y^{M} \cdot \partial Y^{R} \partial Y^{N} \cdot \partial Y^{S}\right) \\
& \left.+h_{M N} G_{P R}\left(\partial Y^{M} \cdot \partial Y^{N} \partial \xi^{P} \cdot \partial Y^{R}-2 \partial Y^{M} \cdot \partial \xi^{P} \partial Y^{N} \cdot \partial Y^{R}\right)\right] .
\end{aligned}
$$


The bulk quantities in (A.1) and (A.2), that is the background metric $G_{M N}$ and the fluctuation $h_{M N}$, are computed in the background brane position. This is because (A.1) and (A.2) come from the variation of the brane action (2.6) where the bulk fields are computed in the brane position.

\section{B Spin-0 bilinear action in the light cone static gauge}

Here we provide the spin-0 action in the light cone static gauge defined by (4.1) and (4.3). This is the only part where the branons $\xi^{\underline{m}}$ appear.

Let us start with the spin-0 action that only depends on the bulk fields. The non vanishing terms are the following:

$$
\begin{aligned}
& S^{(0)}(h, h)=-\frac{1}{4 \kappa^{2}} \int d^{D} X \sqrt{-G}\left[\partial_{\mu} h_{\underline{m n}} \partial^{\mu} h \frac{\underline{m n}}{2}+\partial_{\rho} h_{\underline{m n}} \partial_{\rho} h \frac{m n}{\underline{m n} ;}+h_{\underline{m n}} h^{\underline{m n} ; l}\right. \\
& +h_{\rho \rho}^{2}\left(D_{2} A^{\prime} B^{\prime}+2 A^{\prime \prime}\right)+2\left(A^{\prime \prime}+A^{\prime 2}\right) h_{\rho \rho} h_{i}{ }^{i} \\
& \left(D_{2} A^{\prime} B^{\prime}+2 A^{\prime \prime}-\frac{1}{2} B^{\prime 2}-2 B^{\prime \prime}\right) h_{\rho m} h_{\rho}^{m}-4 A^{\prime} h_{\rho}^{\underline{n}} h_{m \underline{m}}^{; m} \\
& +h_{i}{ }^{i} h_{m}{ }^{m} A^{\prime} B^{\prime}+2\left(B^{\prime \prime}+\frac{B^{\prime 2}}{2}\right) h_{\rho \rho} h_{m}{ }^{m}+\frac{1}{2} B^{\prime 2} h_{m n} h^{m n}+\frac{1}{2} B^{\prime 2}\left(h_{m}^{m}\right)^{2} \\
& -2 e^{-B} h_{l}^{m} h^{n}{ }_{h} \Omega_{m n}{ }^{l h}+2 \kappa^{2} h_{\underline{l m}} h^{\underline{\underline{l}}} \underline{\underline{n}}\left(\frac{1}{2} e^{\phi / 2} F^{\underline{m}}{ }_{\underline{h}} F^{\underline{n h}}+\frac{1}{4 \kappa^{2}} \partial^{\underline{m}} \phi \partial^{\underline{n}} \phi\right) \\
& +\kappa^{2} e^{\phi / 2} h \frac{\underline{m n}}{h} \underline{\underline{l h}} F_{\underline{l m}} F_{\underline{h n}}+\frac{1}{2}\left(\partial_{\mu} h_{i}{ }^{i} \partial^{\mu} h_{j}{ }^{j}+\partial_{\rho} h_{i}{ }^{i} \partial_{\rho} h_{j}{ }^{j}+h_{i ; m}{ }^{i} h_{j}{ }^{j ; m}\right) \\
& \left.+\left(h_{i}{ }^{i}\right)^{2}\left(\frac{1}{2} A^{\prime 2}+\frac{T}{2} \kappa^{2} \sqrt{g / G} \delta\left(X_{c}-Y_{c}\right)\right)\right] \text {, }
\end{aligned}
$$

where $\Omega_{m n h}{ }^{l}$ is the Riemann tensor for the metric $K_{m n}$ and $X_{c}$ and $Y_{c}$ are defined below eq. (4.5). We observe that in the last line of (B.1) there is an explicit brane contribution (the tension of the brane $T$ appears explicitly). Moreover,

$$
\begin{aligned}
S^{(0)}(V, V)= & -\frac{1}{2} \int d^{D} X \sqrt{-G} e^{\phi / 2}\left[\partial_{\mu} V_{\underline{m}} \partial^{\mu} V^{\underline{m}}+D_{\underline{m}} V_{\underline{n}} D^{\underline{m}} V^{\underline{n}}+\left(-2 A^{\prime 2}+\frac{1}{4} \phi^{\prime 2}\right) V_{\rho}^{2}\right. \\
& +\left(-2 A^{\prime}+\phi^{\prime}\right) V_{\rho} D_{\underline{m}} V^{\underline{m}}+R \underline{\underline{m} n} V_{\underline{m}} V_{\underline{n}}+2 \bar{g} F^{\underline{m n}} V_{\underline{m}} \times V_{\underline{n}} \\
& \left.-\frac{1}{2} \phi^{\prime} V_{\rho} D_{m} V^{m}+\frac{1}{2} \phi^{\prime} V^{m} D_{m} V_{\rho}+\kappa^{2} e^{\phi / 2}\left(F_{\underline{l}} \underline{m} V_{\underline{m}}\right) F^{\underline{l n}} V_{\underline{n}}\right] \\
S^{(0)}(h, V)= & \int d^{D} X \sqrt{-G} e^{\phi / 2}\left[F \underline{n \underline{m}} V_{\underline{m}} h_{\underline{l n}}{ }^{; \underline{l}}+\left(D_{\underline{n}} V_{\underline{m}}-D_{\underline{m}} V_{\underline{n}}\right) h_{\underline{\underline{n}}} F^{\underline{l m}}\right. \\
& \left.-\frac{1}{2} A^{\prime} F^{\rho \underline{m}} V_{\underline{m}} h_{i}{ }^{i}+A^{\prime} F^{\underline{m n}} V_{\underline{n}} \underline{h_{m} \rho}\right]
\end{aligned}
$$




$$
\begin{aligned}
& S^{(0)}(\tau, \tau)=-\int d^{D} X \sqrt{-G}\left[\frac{1}{4 \kappa^{2}} \partial_{M} \tau \partial^{M} \tau+\frac{1}{2}\left(\frac{\partial^{2} \mathcal{V}}{\partial \phi^{2}}+\frac{1}{16} e^{\phi / 2} F^{2}+\frac{\phi^{\prime 2}}{4 \kappa^{2}}\right) \tau^{2}\right], \\
& S^{(0)}(h, \tau)=\int d^{D} X \sqrt{-G}\left\{\frac{1}{2 \kappa^{2}}\left[\phi^{\prime} \tau\left(A^{\prime} h_{\rho \rho}+h_{\underline{m} \rho} \underline{\underline{m}}-\frac{1}{2} A^{\prime} h_{i}{ }^{i}\right)+h \underline{\underline{m} \rho} \partial_{\underline{m}} \tau \phi^{\prime}\right]\right. \\
& \left.+\frac{1}{4} e^{\phi / 2} F^{\underline{m l}} F^{\underline{n}}{ }_{\underline{l}} \tau h_{\underline{m n}}\right\} \text {, } \\
& S^{(0)}(V, \tau)=\int d^{D} X \sqrt{-G} e^{\phi / 2}\left[\frac{1}{4} F \frac{m n}{2} \tau\left(D_{\underline{n}} V_{\underline{m}}-D_{\underline{m}} V_{\underline{n}}\right)-\frac{1}{2} \phi^{\prime} F^{\rho m} \tau V_{m}\right], \\
& S^{(0)}\left(V_{2}, V_{2}\right)=-\frac{1}{16} \int d^{D} X \sqrt{-G} e^{\phi}\left\{e^{-2 A} \partial_{M} V_{i j} \partial^{M} V_{i j}\right. \\
& -2 e^{-4 A-2 \phi}\left(e^{\phi+2 A} V^{\underline{n}} \underline{m}\right)_{; \underline{n}}\left(e^{\phi+2 A} V \underline{l m}\right)_{; \underline{l}} \\
& -4 V \underline{\underline{m n}} e^{-A} \partial_{\underline{m}}\left[e^{-A-\phi}\left(e^{\phi+2 A} V \underline{\underline{\underline{n}}}\right)_{; \underline{l}}\right] \\
& \left.+\partial_{\mu} V_{\underline{m n}} \partial^{\mu} V^{\underline{m n}}+\frac{1}{3} V_{[\underline{n l} ; \underline{m}]} V^{[\underline{n l} ; \underline{m}]}+\frac{\kappa^{2}}{2} \gamma^{2} e^{\phi / 2}\left(V_{\underline{m n}} F^{\underline{m n}}\right)^{2}\right\} \\
& S^{(0)}\left(V, V_{2}\right)=-\frac{\kappa}{4} \gamma \int d^{D} X \sqrt{-G} e^{\phi} V_{\underline{m n}} F \underline{\underline{m n}}\left[\left(A^{\prime}+\frac{1}{2} \phi^{\prime}\right) V_{\rho}+D_{\underline{l}} V^{\underline{l}}\right] .
\end{aligned}
$$

We have no mixing of the form $S^{(0)}\left(h, V_{2}\right)$ and $S^{(0)}\left(\tau, V_{2}\right)$ as a consequence of $H_{M N P}=0$ (at the background level). We have checked that the term $S^{(0)}(V, V)$ reduces, as it should, to the corresponding action in ref. [9] in the case in which $V_{\underline{m}}$ is orthogonal to the background gauge field.

Let us consider now the branon-dependent action. This turns out to have the following form: $:^{32}$

$$
S^{(0)}(h, \xi)+S^{(0)}(V, \xi)+S^{(0)}(\tau, \xi)+S^{(0)}(\xi, \xi) .
$$

Therefore, the fields $\xi^{\underline{m}}$ in general couple with some bulk fields, but these mixings are confined to the spin-0 action. The explicit expressions for the different pieces are

$$
\begin{aligned}
S^{(0)}(h, \xi)= & -\frac{T}{2} \int d^{4} x \sqrt{-g}\left[2 \xi^{\underline{m}}\left(A^{\prime} h_{\rho \underline{m}}+h_{\underline{n m}}{ }^{; \underline{n}}\right)+e^{-A} \xi^{\underline{m}} \partial_{\underline{m}} h_{i i}\right], \\
S^{(0)}(V, \xi)= & T \kappa^{2} \int d^{4} x \sqrt{-g} e^{\phi / 2} F_{\underline{m}} \underline{\underline{n}} V_{\underline{n}} \xi^{\underline{m}}, \\
S^{(0)}(\tau, \xi)= & \frac{T}{2} \int d^{4} x \sqrt{-g} \xi^{\underline{m}} \partial_{\underline{m}} \phi \tau, \\
S^{(0)}(\xi, \xi)= & -\frac{T}{2} \int d^{4} x \sqrt{-g}\left[G_{\underline{m n}} \partial_{\mu} \xi^{\underline{m}} \partial^{\mu} \xi^{\underline{n}}+\frac{1}{2} \xi^{\underline{m}} \partial_{\underline{m}} g_{\mu \nu} \xi^{\underline{n}} \partial_{\underline{n}} g_{\eta \sigma}\left(\frac{1}{2} g^{\mu \nu} g^{\eta \sigma}-g^{\mu \eta} g^{\nu \sigma}\right)\right. \\
& \left.+\frac{1}{2} \xi^{\underline{m}} \xi^{\underline{n}} \partial_{\underline{m}} \partial_{\underline{n}} g_{\mu \nu} g^{\mu \nu}+T \kappa^{2} \sqrt{g / G} \delta\left(Y_{c}-Y_{c}\right) \xi^{\underline{m}} \xi_{\underline{m}}\right] .
\end{aligned}
$$

We discuss the various singularities that can be observed in the above in Subsection 4.2 .3 and below.

\footnotetext{
${ }^{32}$ The term of the form $S^{(0)}\left(V_{2}, \xi\right)$ vanishes as a consequence of $H_{M N P}=0$ at the background level, which in turn follows from our background ansatz.
} 


\section{Spin-0 spectrum for 6D supergravity compactification}

We finally analyse the (massive) spin- 0 fluctuations in $6 \mathrm{D}$ braneworlds by using the general spin-0 action given in appendix B. Here we discard the branons. There are different ways to make this truncation consistently, e.g. by introducing an orbifold that projects them out. In ref. [9] such an orbifold has been defined taking into account the presence of at least two patches in the description of spherical topologies. Here we only use the fact that the orbifold action in the intersection of the two patches is $\varphi \rightarrow \varphi+\pi$. In the absence of the branons the $\delta(0)$ singularities mentioned in Subsection 4.2 .3 obviously disappear. We shall see that it is also possible to deal with the other type of singularities mentioned there and extract a finite spectrum.

Here we focus on the unwarped solutions and in particular on the rugbyballs and saddlespheres defined in Subsection 2.3. In this case we will be able to generalize the harmonic analysis developed in Subsection 5.2.2 to the spin-0 sector, which involves 2D tensors as well as $2 \mathrm{D}$ vectors and scalars. This technique allows us to transform complicated coupled differential equations into algebraic equations whose solutions can be found exactly. The relevant fluctuations are $h_{m n}, V_{m}, \tau, V_{i j}$ and $V_{m n}$, where $m$ and $n$ run over $\theta$ and $\varphi$. We observe that the fluctuations $V_{m}$ orthogonal to background gauge field decouple to the other fields and have already been analyzed in [9]; therefore here we assume $V_{m}$ to be parallel to the background gauge field. One should keep in mind that, if the branons are projected out by the above-mentioned orbifold, only the modes with $\mathbf{m}$ even survive (in the Fourier expansion over $\left.e^{i \mathbf{m} \varphi}\right)$. The spin-0 action in the light cone gauge assumes the following form:

$$
\begin{aligned}
S^{(0)}(h, h)= & -\frac{1}{4 \kappa^{2}} \int d^{6} X \sqrt{-G}\left[\partial_{\mu} h_{m n} \partial^{\mu} h^{m n}+h_{m n ; l} h^{m n ; l}\right. \\
& -2 h^{m} h^{n}{ }_{h} R_{m n}{ }^{l h}+\kappa^{2} h_{l m} h^{l}{ }_{n} F^{m}{ }_{h} F^{n h}+\kappa^{2} h^{m n} h^{l h} F_{l m} F_{h n} \\
& \left.-\frac{1}{2} h_{m}{ }^{m}\left(\partial^{2}+D^{2}\right) h_{n}{ }^{n}+\frac{T}{2} \kappa^{2}\left(h_{m}{ }^{m}\right)^{2} \sqrt{g / G} \delta\left(X_{2}-Y_{2}\right)\right], \\
S^{(0)}(V, V)= & -\frac{1}{2} \int d^{6} X \sqrt{-G}\left[\partial_{\mu} V_{m} \partial^{\mu} V^{m}+V_{m ; n} V^{m ; n}+\frac{1}{2} R V_{m} V^{m}+\frac{\kappa^{2}}{2} F^{2} V_{m} V^{m}\right], \\
S^{(0)}(\tau, \tau)= & \frac{1}{4 \kappa^{2}} \int d^{6} X \sqrt{-G}\left[\tau\left(\partial^{2}+D^{2}-\frac{4}{r_{0}^{2}}\right) \tau\right], \\
S^{(0)}\left(V_{2}, V_{2}\right)= & \int d^{6} X \sqrt{-G}\left[\frac{1}{16} V_{i j}\left(\partial^{2}+D^{2}\right) V_{i j}+\frac{1}{16} V_{m n}\left(\partial^{2}+D^{2}\right) V^{m n}-\frac{\kappa^{2} \gamma^{2}}{32}\left(V_{m n} F^{m n}\right)^{2}\right], \\
S^{(0)}(h, V)= & \int d^{6} X \sqrt{-G}\left[-V_{n ; m} h_{l}{ }^{n} F^{l m}\right], \\
S^{(0)}(h, \tau)= & \frac{1}{8} \int d^{6} X \sqrt{-G} F^{2} \tau h_{m}{ }^{m}, \\
S^{(0)}(V, \tau)= & \int d^{6} X \sqrt{-G} \frac{\tau}{4} F^{m n}\left(V_{m ; n}-V_{n ; m}\right), \\
S^{(0)}\left(V, V_{2}\right)= & -\frac{\kappa}{4} \gamma \int d^{6} X \sqrt{-G} V_{m n} F^{m n} V_{l} ; l,
\end{aligned}
$$


where we have used the light cone gauge relation $h_{i}{ }^{i}+h_{m}{ }^{m}=0$ in $S^{(0)}(h, h)$ and the property $F^{m l} F_{l}^{n}=G^{m n} F^{2} / 2$ in $S^{(0)}(V, V)$ and $S^{(0)}(h, \tau)$, which is a consequence of (5.20).

We now want to use a technique similar to that explained in the spin-1 sector, in order to transform the above differential problem into an algebraic one. Note that the method provided in Subsection 5.2.2 can be already applied to perform this transformation in the terms $S^{(0)}(V, V), S^{(0)}(\tau, \tau), S^{(0)}\left(V_{2}, V_{2}\right), S^{(0)}(V, \tau)$ and $S^{(0)}\left(V, V_{2}\right)$ as they only involve $2 \mathrm{D}$ scalars and $2 \mathrm{D}$ vectors. ${ }^{33}$ What we have done there is to identify appropriate masssquared operators from the diagonal part of the bilinear action, which are Hermitian once the HCs are imposed. In this way we were able to define complete sets of $2 \mathrm{D}$ scalar and vector harmonics. Then we focused on the cases in which the derivative relation, eq. (5.32), between scalar and vector harmonics holds. That relation is what allowed us to deal with the derivative couplings between scalars and vectors and transform the spin-1 differential problem into an algebraic one, which could easily be solved.

Here we generalize the above procedure to include the $2 \mathrm{D}$ tensor fluctuations in $h_{m n}$. Indeed, $h_{m n}$ can be decomposed into its trace, $h_{m}^{m}$, and traceless part, $\tilde{h}_{m n} \equiv h_{m n}-$ $G_{m n} h_{l}^{l} / 2$, so that the first entry in (C.1) decomposes into the two terms:

$$
\begin{aligned}
& S^{(0)}\left(h_{m}{ }^{m}, h_{m}{ }^{m}\right)=\frac{1}{8 \kappa^{2}} \int d^{6} X \sqrt{-G}\left[h_{m}{ }^{m}\left(\partial^{2}+D^{2}-\frac{\kappa^{2}}{4} F^{2}\right) h_{n}{ }^{n}\right], \\
& S^{(0)}\left(\tilde{h}_{m n}, \tilde{h}_{m n}\right)=\frac{1}{4 \kappa^{2}} \int d^{6} X \sqrt{-G}\left[\tilde{h}_{m n}\left(\partial^{2}+D^{2}-R\right) \tilde{h}^{m n}\right],
\end{aligned}
$$

where we used the following identities:

$$
R_{p m q n}=\frac{R}{2}\left(G_{p q} G_{m n}-G_{m q} G_{p n}\right), \quad F_{l m} F_{h n}=\frac{F^{2}}{2}\left(G_{l h} G_{m n}-G_{m h} G_{l n}\right) .
$$

Observe that $h_{m}{ }^{m}$ is in fact a 2D scalar field, and we can expand it in terms of the 2D scalar harmonics found in Subsection 5.1.2. The fluctuations $\tilde{h}_{m n}$ are instead genuine 2D tensor fluctuations, and the appropriate mass-squared operator is $-D^{2}+R$. Thus, we would like to solve the eigenproblem:

$$
\left(-D^{2}+R\right) \tilde{h}_{m n}=\mu_{T}^{2} \tilde{h}_{m n},
$$

with the given NCs and HCs, where $\mu_{T}^{2}$ are the corresponding mass-eigenvalues.

In a general basis, eq. (C.4) is a set of two coupled differential equations (the traceless property removes one out of the three components of a rank two symmetric tensor in two dimensions). However, by writing down eq. (C.4) in the \pm basis defined in (5.29):

$$
\left(-D^{2}+R\right) h_{ \pm \pm}=\mu_{T}^{2} h_{ \pm \pm}
$$

where we used $h_{ \pm \pm}=\tilde{h}_{ \pm \pm}$, and by explicitly evaluating $D^{2} h_{ \pm \pm}$, one finds that the equations for $h_{++}$and $h_{--}$are decoupled, like those of the $h_{+i}$ and $h_{-i}$ fields in the spin-1 sector. After a long but straightforward calculation we find

$$
-\partial_{\theta}^{2} f^{ \pm \pm}+\frac{\dot{B}}{2} \partial_{\theta} f^{ \pm \pm}+\left(\mathbf{m}^{2} e^{-B} \pm 2 \mathbf{m} \dot{B} e^{-B / 2}+\frac{\dot{B}^{2}}{2}-\frac{\ddot{B}}{2}\right) f^{ \pm \pm}=\frac{r_{0}^{2}}{4} \mu_{T}^{2} f^{ \pm \pm},
$$

\footnotetext{
${ }^{33}$ The analysis of the $V_{m n}$-EOMs shows that $V_{m n} / \sqrt{-G}$ is a $2 \mathrm{D}$ scalar.
} 
where a dot represents a derivative with respect to $\theta$ and $f^{ \pm \pm}$is the wave function of $h_{ \pm \pm}$, defined by a KK expansion

$$
h_{ \pm \pm}(X)=\sum_{\mathbf{n}, \mathbf{m}} h_{ \pm \pm \mathbf{n m}}(x) f_{\mathbf{n m}}^{ \pm \pm}(\theta) e^{i \mathbf{m} \varphi}, \quad \text { with } \mathbf{m}=\text { generic integer }
$$

and in (C.6) the KK numbers $\mathbf{n}$ and $\mathbf{m}$ are understood. The eigenvalues $\mu_{T}^{2}$ can be found by using the technique discussed in ref. [6]: one can put the equations into the hypergeometric form, consider the general solution to the hypergeometric equation and then impose the HCs and NCs. We find

- For $|\mathbf{m}| \omega \geq 2$

$$
\mu_{T}^{2}=\frac{4}{r_{0}^{2}}[(\mathbf{n}+|\mathbf{m}| \omega)(\mathbf{n}+|\mathbf{m}| \omega+1)-2]
$$

- For $-2<\mathbf{m} \omega<2$

$$
\mu_{T}^{2}=\frac{4}{r_{0}^{2}}[(\mathbf{n}+2)(\mathbf{n}+3)-2]
$$

where $\mathbf{n}=0,1,2,3, \ldots$ In this way we have found a complete set of $2 \mathrm{D}$ tensor harmonics.

We now remember that, in the spin-1 sector analysed in Subsection 5.2.2, one can generate the $2 \mathrm{D}$ vector harmonics by acting with derivatives over the $2 \mathrm{D}$ scalar harmonics (see eq. (5.32) and the discussion right above). We can imagine that something similar happens here and the 2D tensor harmonics (C.5) can be generated by acting with derivatives over $2 \mathrm{D}$ vector harmonics. This is indeed the case and in order to see it let us consider the $2 \mathrm{D}$ vector harmonics for $V_{m}$ :

$$
\left(-D^{2}+\frac{R}{2}\right) V_{m}=\mu_{V}^{2} V_{m}
$$

where $\mu_{V}^{2}$ are the $2 \mathrm{D}$ vector mass-eigenvalues. From now on we shall assume Condition (5.31), so that $\mu_{V}^{2}=\mu^{2}$, with $\mu^{2}$ the $2 \mathrm{D}$ scalar mass-eigenvalues given in (5.28). After some manipulation it is easy to show that if $V_{m}$ satisfies the previous equation then we also have

$$
-D^{2} \tilde{V}_{m ; n}+R\left(\tilde{V}_{m ; n}+\tilde{V}_{n ; m}\right)+\frac{1}{2}\left(R_{; m} V_{n}+R_{; n} V_{m}-G_{m n} R_{; l} V^{l}\right)=\mu^{2} \tilde{V}_{m ; n}
$$

where $\tilde{V}_{m ; n} \equiv V_{m ; n}-G_{m n} V_{l}^{; l} / 2$. This equation is valid for any unwarped compactification, but in the rugbyball case it can be simplified. Although the Ricci scalar is not constant everywhere like in the sphere limit as it contains delta-functions, these additional delta function terms can be discarded in eq. (C.11) because they are dominated by stronger singularities, ${ }^{34}$ which emerge from $D^{2} \tilde{V}_{m ; n}$. This allows us to write (C.11) as follows:

$$
-D^{2} \tilde{V}_{m ; n}+R_{s}\left(\tilde{V}_{m ; n}+\tilde{V}_{n ; m}\right)=\mu^{2} \tilde{V}_{m ; n},
$$

where $R_{s}$ is the Ricci scalar of the sphere $\left(R_{s}=8 / r_{0}^{2}\right)$, or, in the \pm basis,

$$
\left(-D^{2}+2 R_{s}\right) V_{ \pm ; \pm}=\mu^{2} V_{ \pm ; \pm},
$$

\footnotetext{
${ }^{34}$ This is a quite generic property of rugbyball compactifications $[6,9]$.
} 
where we used $V_{ \pm ; \pm}=\tilde{V}_{ \pm ; \pm}$. Now, comparing the eigenproblems for $h_{ \pm \pm}$and $V_{ \pm ; \pm}$, eqs. (C.5) and (C.13), we see that their eigenfunctions will belong to the same orthogonal set provided that:

$$
\mu_{T}^{2}=\mu^{2}-R_{s}=\mu^{2}-8 / r_{0}^{2} .
$$

By comparing the $2 \mathrm{D}$ vector mass-eigenvalues, $\mu^{2}$ given in (5.28), with the $2 \mathrm{D}$ tensor eigenvalues, $\mu_{T}^{2}$ given in eqs. (C.8) and (C.9), we find that Condition (C.14) is indeed true in the following cases:

- For $\mathbf{m}=0$ or $|\mathbf{m}| \geq 2 / \omega$, which we denote by $0 \nless|\mathbf{m}| \omega \nless 2$, with the constraint $\{\mathbf{n}, \mathbf{m}\} \neq\{0,0\},\{1,0\}$.

- For $|\mathbf{m}| \omega=1$, with the constraint $\mathbf{n} \neq 0$.

- The sphere case $(\omega=1)$, with the constraint $\{\mathbf{n}, \mathbf{m}\} \neq\{0,0\},\{1,0\},\{0, \pm 1\}$. This result is in agreement with that obtained by using the Wigner functions [31].

When (C.14) is true a derivative relation between the $2 \mathrm{D}$ tensor and $2 \mathrm{D}$ vector wave functions holds:

$$
D_{ \pm}\left(f_{\mathbf{n m}}^{ \pm}(\theta) e^{i \mathbf{m} \varphi}\right)=c_{T \mathbf{n m}} f_{\mathbf{n m}}^{ \pm \pm}(\theta) e^{i \mathbf{m} \varphi},
$$

where $c_{T} \mathbf{n m}$ are normalization constants which, having chosen a convenient normalization for the wave functions, can be fixed to be $c_{T \mathbf{n} \mathbf{m}}=\sqrt{\mu_{T \mathbf{n} \mathbf{m}}^{2}} / \sqrt{2}$.

It remains to expand the $6 \mathrm{D}$ fields in the action (C.1) into their harmonics on the rugbyball and integrate over the extra dimensions. Thanks to the derivative relations (5.32), (C.15), and $F^{2}=$ const, the mass-squared operator reduces to an algebraic matrix with constant entries, which can easily be diagonalized. We note that the massmatrix turns out to be well-defined despite the singularities mentioned in Subsection 4.2.3.

We end with the resulting spectrum for spin-0 fields (which can be trusted when eq. (C.14) holds). For definiteness we focus here on the 6D supergravity setup, but there are no problems in deriving the squared masses in the EYM $\Lambda$ case as well. We split the spectrum according to the values of $l \equiv \mathbf{n}+|\mathbf{m}| \omega$ :

- For $l=0$

$$
\frac{r_{0}^{2}}{4} M^{2}=0,0,2,[2]
$$

- For $l=1$

$$
\frac{r_{0}^{2}}{4} M^{2}=2,6,[2],[2],[2],[6]
$$

- For $l>1$

$$
\begin{aligned}
& M^{2}=\mu_{\mathbf{n} \mathbf{m}}^{2} \quad \text { with multiplicity } 1[+3] \\
& M^{2}=\frac{4}{r_{0}^{2}}\left(1+\frac{r_{0}^{2}}{4} \mu_{\mathbf{n} \mathbf{m}}^{2}-\sqrt{1+r_{0}^{2} \mu_{\mathbf{n} \mathbf{m}}^{2}}\right) \text { with multiplicity } 1[+1] \\
& M^{2}=\frac{4}{r_{0}^{2}}\left(1+\frac{r_{0}^{2}}{4} \mu_{\mathbf{n} \mathbf{m}}^{2}+\sqrt{1+r_{0}^{2} \mu_{\mathbf{n} \mathbf{~}}^{2}}\right) \text { with multiplicity } 1[+1]
\end{aligned}
$$


where the square brackets denote helicity- 0 components of higher spin fields and the remaining modes are physical spin-0 fields. We observe that there are neither ghosts nor tachyons and we recover the two massless fields discussed in Subsection 5.4.2.

As an effective check of the above spectrum we observe that it correctly reduces, when $\omega=1$, to the sphere result obtained by directly expanding the bulk fields over the Wigner functions [27].

\section{References}

[1] L. Randall and R. Sundrum, A large mass hierarchy from a small extra dimension, Phys. Rev. Lett. 83 (1999) 3370 [hep-ph/9905221] [SPIRES].

[2] L. Randall and R. Sundrum, An alternative to compactification, Phys. Rev. Lett. 83 (1999) 4690 [hep-th/9906064] [SPIRES].

[3] H. Nishino and E. Sezgin, Matter and gauge couplings of $N=2$ supergravity in six-dimensions, Phys. Lett. B 144 (1984) 187 [SPIRES].

[4] G.W. Gibbons, R. Güven and C.N. Pope, 3-branes and uniqueness of the Salam-Sezgin vacuum, Phys. Lett. B 595 (2004) 498 [hep-th/0307238] [SPIRES].

[5] Y. Aghababaie et al., Warped brane worlds in six dimensional supergravity, JHEP 09 (2003) 037 [hep-th/0308064] [SPIRES].

[6] S.L. Parameswaran, S. Randjbar-Daemi and A. Salvio, Gauge fields, fermions and mass gaps in 6D brane worlds, Nucl. Phys. B 767 (2007) 54 [hep-th/0608074] [SPIRES].

[7] C.P. Burgess, C. de Rham, D. Hoover, D. Mason and A.J. Tolley, Kicking the rugby ball: perturbations of $6 D$ gauged chiral supergravity, JCAP 02 (2007) 009 [hep-th/0610078] [SPIRES].

[8] H.M. Lee and A. Papazoglou, Gravitino in six-dimensional warped supergravity, Nucl. Phys. B 792 (2008) 166 [arXiv:0705.1157] [SPIRES].

[9] S.L. Parameswaran, S. Randjbar-Daemi and A. Salvio, Stability and negative tensions in $6 D$ brane worlds, JHEP 01 (2008) 051 [arXiv:0706.1893] [SPIRES].

[10] C.P. Burgess, S.L. Parameswaran and I. Zavala, The fate of unstable gauge flux compactifications, arXiv:0812.3902 [SPIRES].

[11] H.M. Lee and A. Papazoglou, Scalar mode analysis of the warped Salam-Sezgin model, Nucl. Phys. B 747 (2006) 294 [Erratum ibid. 765 (2007) 200] [hep-th/0602208] [SPIRES].

[12] S. Randjbar-Daemi and M. Shaposhnikov, A formalism to analyze the spectrum of brane world scenarios, Nucl. Phys. B 645 (2002) 188 [hep-th/0206016] [SPIRES].

[13] R. Sundrum, Compactification for a three-brane universe, Phys. Rev. D 59 (1999) 085010 [hep-ph/9807348] [SPIRES].

[14] See for e.g., M. Bando, T. Kugo, T. Noguchi and K. Yoshioka, Brane fluctuation and suppression of Kaluza-Klein mode couplings, Phys. Rev. Lett. 83 (1999) 3601 [hep-ph/9906549] [SPIRES];

N. Arkani-Hamed, S. Dimopoulos and G.R. Dvali, Phenomenology, astrophysics and cosmology of theories with sub-millimeter dimensions and TeV scale quantum gravity, Phys. Rev. D 59 (1999) 086004 [hep-ph/9807344] [SPIRES]; 
A. Dobado and A.L. Maroto, The dynamics of the Goldstone bosons on the brane, Nucl. Phys. B 592 (2001) 203 [hep-ph/0007100] [SPIRES];

H. Murayama and J.D. Wells, Graviton emission from a soft brane, Phys. Rev. D 65 (2002) 056011 [hep-ph/0109004] [SPIRES].

[15] R. Sundrum, Effective field theory for a three-brane universe, Phys. Rev. D 59 (1999) 085009 [hep-ph/9805471] [SPIRES].

[16] S. Randjbar-Daemi, A. Salam, E. Sezgin and J.A. Strathdee, An anomaly free model in six-dimensions, Phys. Lett. B 151 (1985) 351 [SPIRES].

[17] S.D. Avramis, A. Kehagias and S. Randjbar-Daemi, A new anomaly-free gauged supergravity in six dimensions, JHEP 05 (2005) 057 [hep-th/0504033] [SPIRES];

S.D. Avramis and A. Kehagias, A systematic search for anomaly-free supergravities in six dimensions, JHEP 10 (2005) 052 [hep-th/0508172] [SPIRES];

R. Suzuki and Y. Tachikawa, More anomaly-free models of six-dimensional gauged supergravity, J. Math. Phys. 47 (2006) 062302 [hep-th/0512019] [SPIRES].

[18] H. Nishino and E. Sezgin, The complete $N=2, D=6$ supergravity with matter and Yang-Mills couplings, Nucl. Phys. B 278 (1986) 353 [SPIRES].

[19] G.W. Gibbons and S.W. Hawking, Action integrals and partition functions in quantum gravity, Phys. Rev. D 15 (1977) 2752 [SPIRES];

H.A. Chamblin and H.S. Reall, Dynamic dilatonic domain walls, Nucl. Phys. B 562 (1999) 133 [hep-th/9903225] [SPIRES].

[20] Z. Lalak and R. Matyszkiewicz, Boundary terms in brane worlds, JHEP 11 (2001) 027 [hep-th/0110141] [SPIRES].

[21] S.M. Carroll and M.M. Guica, Sidestepping the cosmological constant with football-shaped extra dimensions, hep-th/0302067 [SPIRES];

I. Navarro, Codimension two compactifications and the cosmological constant problem, JCAP 09 (2003) 004 [hep-th/0302129] [SPIRES].

[22] R. Sundrum, Compactification for a three-brane universe, Phys. Rev. D 59 (1999) 085010 [hep-ph/9807348] [SPIRES];

J.-W. Chen, M.A. Luty and E. Ponton, A critical cosmological constant from millimeter extra dimensions, JHEP 09 (2000) 012 [hep-th/0003067] [SPIRES].

[23] M. Kaku, Ghost-free formulation of quantum gravity in the light cone gauge, Nucl. Phys. B 91 (1975) 99 [SPIRES].

[24] C.A. Lütken and C.R. Ordonez, Vacuum energy of eleven-dimensional supergravity, Class. Quant. Grav. 4 (1987) 1543 [SPIRES].

[25] S. Randjbar-Daemi, A. Salam and J.A. Strathdee, Towards a selfconsistent computation of vacuum energy in eleven-dimensional supergravity, Nuovo Cim. B 84 (1984) 167 [SPIRES].

[26] S. Randjbar-Daemi and M.H. Sarmadi, Graviton induced compactification in the light cone gauge, Phys. Lett. B 151 (1985) 343 [SPIRES].

[27] For explicit computations in the spherical case, see the Appendix C of the Ph.D. thesis, A. Salvio, Aspects of physics with two extra dimensions, hep-th/0701020 [SPIRES]. 
[28] E.A. Mirabelli and M.E. Peskin, Transmission of supersymmetry breaking from a 4-dimensional boundary, Phys. Rev. D 58 (1998) 065002 [hep-th/9712214] [SPIRES]; S. Ichinose and A. Murayama, The $\delta(0)$ singularity in the warped Mirabelli-Peskin model, hep-th/0606167 [SPIRES].

[29] See for e.g., W.D. Goldberger and M.B. Wise, Bulk fields in the Randall-Sundrum compactification scenario, Phys. Rev. D 60 (1999) 107505 [hep-ph/9907218] [SPIRES]; C. Csáki, M. Graesser, L. Randall and J. Terning, Cosmology of brane models with radion stabilization, Phys. Rev. D 62 (2000) 045015 [hep-ph/9911406] [SPIRES];

C. Charmousis, R. Gregory and V.A. Rubakov, Wave function of the radion in a brane world, Phys. Rev. D 62 (2000) 067505 [hep-th/9912160] [SPIRES].

[30] H. Nicolai and C. Wetterich, On the spectrum of Kaluza-Klein theories with noncompact internal spaces, Phys. Lett. B 150 (1985) 347 [SPIRES];

G.W. Gibbons and D.L. Wiltshire, Space-time as a membrane in higher dimensions, Nucl. Phys. B 287 (1987) 717 [hep-th/0109093] [SPIRES];

A. Kehagias, On non-compact compactifications with brane worlds, hep-th/9911134 [SPIRES].

[31] S. Randjbar-Daemi, A. Salam and J.A. Strathdee, Spontaneous compactification in six-dimensional Einstein-Maxwell theory, Nucl. Phys. B 214 (1983) 491 [SPIRES].

[32] A. Salam and E. Sezgin, Chiral compactification on minkowski $X S^{2}$ of $N=2$ Einstein-Maxwell supergravity in six-dimensions, Phys. Lett. B 147 (1984) 47 [SPIRES].

[33] S. Randjbar-Daemi, A. Salvio and M. Shaposhnikov, On the decoupling of heavy modes in Kaluza-Klein theories, Nucl. Phys. B 741 (2006) 236 [hep-th/0601066] [SPIRES].

[34] S. Randjbar-Daemi, A. Salam and J.A. Strathdee, Instability of higher dimensional Yang-Mills systems, Phys. Lett. B 124 (1983) 345 [Erratum ibid. 144 (1984) 455] [SPIRES]; G.R. Dvali, S. Randjbar-Daemi and R. Tabbash, The origin of spontaneous symmetry breaking in theories with large extra dimensions, Phys. Rev. D 65 (2002) 064021 [hep-ph/0102307] [SPIRES].

[35] G.W. Gibbons and C.N. Pope, Consistent $S^{2}$ Pauli reduction of six-dimensional chiral gauged Einstein-Maxwell supergravity, Nucl. Phys. B 697 (2004) 225 [hep-th/0307052] [SPIRES].

[36] M.L. Graesser, J.E. Kile and P. Wang, Gravitational perturbations of a six dimensional self-tuning model, Phys. Rev. D 70 (2004) 024008 [hep-th/0403074] [SPIRES];

M. Peloso, L. Sorbo and G. Tasinato, Standard $4 D$ gravity on a brane in six dimensional flux compactifications, Phys. Rev. D 73 (2006) 104025 [hep-th/0603026] [SPIRES]. 